Les différentes techniques instrumentales 
Collection SFN 10 (2010) 357-378

(C) Owned by the authors, published by EDP Sciences, 2010

DOI: $10.1051 / \mathrm{sfn} / 2010005$

\title{
La diffusion inélastique des neutrons sur monocristal. Le spectromètre 3-axes
}

\author{
B. Hennion
}

Laboratoire Léon Brillouin, CNRS-CEA, CEN-Saclay, 91191 Gif-sur-Yvette Cedex, France

Résumé. Le spectromètre 3-axes est destiné aux mesures de diffusion inélastique des neutrons sur monocristal. Le principe de cette technique développée près des réacteurs à flux continu, son évolution et son utilisation sont présentés. Les effets de résolution d'appareil sont précisés et les méthodes d'analyse indiquées.

\section{INTRODUCTION}

Les propriétés de la matière condensée sont déduites d'observations macroscopiques. Si on cherche à comprendre les mécanismes microscopiques qui les induisent, la première étape consiste à déterminer l'organisation structurale du composé étudié. C'est ce qui est réalisé grâce aux techniques de diffraction, qui permettent de déterminer la position des atomes et éventuellement les caractéristiques magnétiques de la matière solide.

L'étape suivante vise à déterminer les quantités (constantes de force, interactions électroniques et magnétiques, etc...) qui expliquent la structure observée et sont responsables de l'évolution du système: mouvements atomiques, évolution des propriétés magnétiques ou électriques. Ces interactions interviennent en effet dans les propriétés dynamiques du système: réponse à une perturbation, évolution avec la température, qui sont décrites par une susceptibilité qu'on utilise souvent dans l'espace $(\vec{Q}, \omega)$ conjugé de l'espace $(\vec{r}, t)$ : réponse diélectrique, susceptibilité électronique et magnétique. Dans un cristal, ces susceptibilités présentent des singularités correspondant aux excitations élémentaires: les phonons pour les mouvements atomiques, les ondes de spins (ou magnons) pour la dynamique de spin d'un système magnétique.

Rappel (non exhaustif) de quelques thématiques de recherche concernées par ce type d'étude:

\section{- dynamique de réseau}

- courbes de dispersion de phonon pour modélisation

- transition de phases

- interaction électron-phonon

- interaction spin-réseau

\section{- dynamique de spin}

- courbes de dispersion de magnon pour modélisation

- supraconductivité à haute température critique

- magnétorésistance géante

- systèmes magnétiques de basse dimensionnalité.

This is an Open Access article distributed under the terms of the Creative Commons Attribution-Noncommercial License 3.0, which permits unrestricted use, distribution, and reproduction in any noncommercial medium, provided the original work is properly cited. 
La diffusion inélastique des neutrons est un des outils qui permettent d'obtenir des résultats dans ces domaines, car la section efficace de diffusion est directement reliée à la susceptibilité généralisée, de réseau pour les phonons et de spin (pour les magnons). Par contre elle ne fournit aucun accès direct à la susceptibilité électronique.

Pour les études sur monocristaux, où ces susceptibilités s'appuient sur les courbes de dispersion $\omega=f(\vec{q})$ des excitations élémentaires, l'instrument de base est le spectromètre 3-axes.

Nous allons, dans les sections suivantes, décrire le principe de la mesure, puis faire une revue de détail des paramètres instrumentaux qu'il faut adapter à l'objectif visé et enfin évoquer les techniques d'analyse des résultats.

\section{PRINCIPE DE LA MESURE SUR SPECTROMÈTRE 3-AXES}

La mesure est décrite dans l'espace réciproque associé à la structure cristalline de l'échantillon. Lorsqu'on envoie des neutrons sur un échantillon, ils sont diffusés en fonction de la section efficace de diffusion élastique (pas de changement d'énergie) et inélastique (changement d'énergie) des neutrons. Pour avoir une détermination précise des processus de diffusion, on calibre les neutrons avant et après l'échantillon. Pour cela, on interpose sur le trajet des neutrons des monocristaux en position de réflexion de Bragg pour une longueur d'onde donnée $\lambda_{i}$ pour le monochromateur, $\lambda_{f}$ pour l'analyseur, soit en vecteur d'onde: $k_{i}=2 \pi / \lambda_{i}$ et $k_{f}=2 \pi / \lambda_{f}$. Si on met un détecteur après l'analyseur, les neutrons détectés auront échangé du moment et de l'énergie avec l'échantillon, en fonction du bilan (conservation du moment et de l'énergie) :

$$
\left\{\begin{array}{l}
\hbar \vec{k}_{i}-\hbar \vec{k}_{f}=\hbar \vec{Q} \\
\frac{\hbar^{2} k_{i}^{2}}{2 M_{n}}-\frac{\hbar^{2} k_{f}^{2}}{2 M_{n}}=\hbar \omega
\end{array}\right.
$$

avec une intensité fonction de $S(\vec{Q}, \omega)$, la fonction de diffusion des neutrons, elle même directement reliée à la partie imaginaire des susceptibilités de réseau et de spin du système. Si l'échantillon possède la symétrie de translation, on peut décomposer le transfert de moment en $\vec{Q}=\vec{\tau}+\vec{q}$, où $\vec{q}$ appartient à la première zone de Brillouin.

$$
\begin{aligned}
& \text { L'élément clé pour concevoir la mesure est: } \\
& \text { à une position du spectromètre, échantillon compris, correspond un couple } \vec{Q}, \omega \\
& \text { soit } \vec{q}, \omega \text { dans un monocristal où } \vec{Q}=\vec{\tau}+\vec{q} .
\end{aligned}
$$

Bien sûr, la réalité est un peu plus complexe... Nous avons implicitement fait référence à la diffusion cohérente des neutrons, mais il existe aussi une diffusion incohérente dont l'intensité dépend de la composition isotopique de l'échantillon et éventuellement de son désordre structural. Cette diffusion se traduit par un bruit de fond, généralement modulé en $\omega$ comme la densité d'états des excitations et dépendant de $Q$ en fonction de son origine.

Dans certains cas, la diffusion incohérente est tellement forte (cas de l'hydrogène) qu'une substitution isotopique est pratiquement obligatoire pour obtenir un résultat.

Pour un système ordonné, les propriétés dynamiques sont reliées aux excitations élémentaires, qui correspondent à des relations $\omega=f(\vec{q})$. Une mesure sur un spectromètre 3-axes consiste à définir une trajectoire dans l'espace $(\vec{Q}, \omega)$, qu'on parcourt pas à pas, en comptant à nombre de neutrons incidents constant (défini par un moniteur). Si la trajectoire coupe une des lignes $\omega=f(\vec{q})$, on observera un pic d'intensité. On peut ainsi reconstituer point à point la loi $\omega=f(\vec{q})$. L'intensité mesurée est directement reliée à la partie imaginaire de la susceptibilité. 

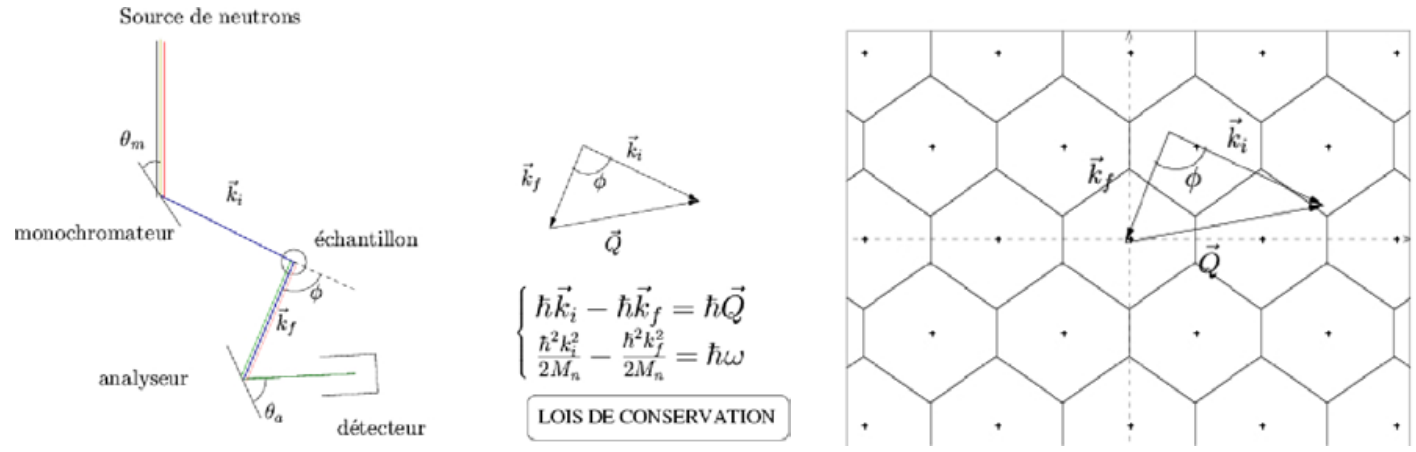

Figure 1. Principe du spectromètre 3-axes: à une position du spectromètre correspond un couple $\vec{Q}$ et $\omega$, soit $\vec{q}$ et $\omega$ dans un monocristal.

En raison des caractéristiques des cristaux (distance interréticulaire, mosaïsicité, courbure horizontale et/ou verticale du monochromateur et de l'analyseur, qualité cristalline de l'échantillon) et de la définition du faisceau (collimateurs), la mesure ne se fait pas en un point $\vec{Q}_{0}, \omega_{0}$ parfaitement défini. On intègre sur une distribution $\vec{Q}, \omega$ centrée sur $\vec{Q}_{0}, \omega_{0}$ et pondérée par la fonction de résolution du spectromètre, qui dépend de tous les écarts à la mesure idéale.

En prenant en compte les caractéristiques du spectromètre, on peut tirer de la mesure: la valeur $(\vec{Q}, \omega)$ de l'excitation, son amortissement éventuel si la largeur mesurée est supérieure à la largeur instrumentale, et son poids.

\section{REMARQUE :}

Comme l'espace réciproque est à 4 dimensions, il est impératif d'avoir des prévisions raisonnables avant d'entamer une mesure... On a a priori un large choix de conditions expérimentales pour effectuer une mesure : choix du monochromateur et de l'analyseur, choix des courbures, choix des collimations, choix de $k_{i}$ ou $k_{f} \ldots$ Il faut trouver un compromis entre la précision requise pour atteindre la physique visée et l'obtention d'un signal raisonnable dans le temps de faisceau disponible, compte tenu du volume de l'échantillon.

\section{CHOIX DE LA SOURCE POUR UNE MESURE}

Suivant le domaine de physique concerné, on peut prévoir le domaine d'énergie des excitations qu'on souhaite observer. Il correspond essentiellement à celui des phénomènes qui peuvent être activés thermiquement. Rappelons les correspondances entre unités : $300 \mathrm{~K} \approx 25 \mathrm{meV} \approx 6 \mathrm{THz} \approx 200 \mathrm{~cm}^{-1}$. L'énergie du neutron est proportionnelle au carré de son vecteur d'onde. Un neutron de longueur d'onde $\lambda=2 \AA$, donc de vecteur d'onde $k=\pi \AA^{-1}$, a une énergie de $20.4 \mathrm{meV}\left(4.93 \mathrm{THz}, 237 \mathrm{~K}, 164 \mathrm{~cm}^{-1}\right)$. Nous utiliserons, de façon arbitraire, le THz dans nos exemples.

Les spectromètres 3-axes sont installés sur des sources de neutron dont la distribution de flux est fonction de la température du modérateur. Le premier choix concerne donc la définition de la source: thermique, froide ou chaude.

- source thermique : La source de base où les neutrons émis par le coeur du réacteur sont ralentis par collisions en traversant une épaisseur d'eau lourde calibrée pour fournir un maximum de neutrons pour un vecteur d'onde $\approx k_{i}=4.1 \AA^{-1}$. Cette source permet des mesures dans une gamme de transfert d'énergie de 1 à $\approx 30 \mathrm{THz}$ ( 1 à $\approx 120 \mathrm{meV}$ ) (IN8, IN20, IN22 à l'ILL, 1T et $2 \mathrm{~T}$ au LLB). 


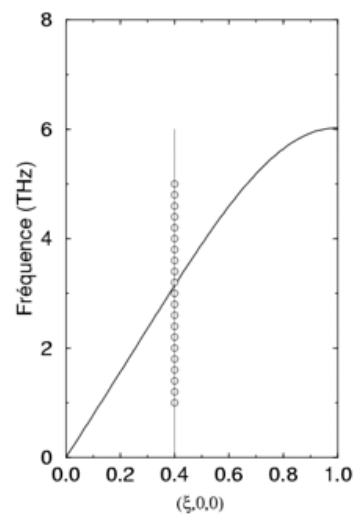

mesure à Q constant et kf constant
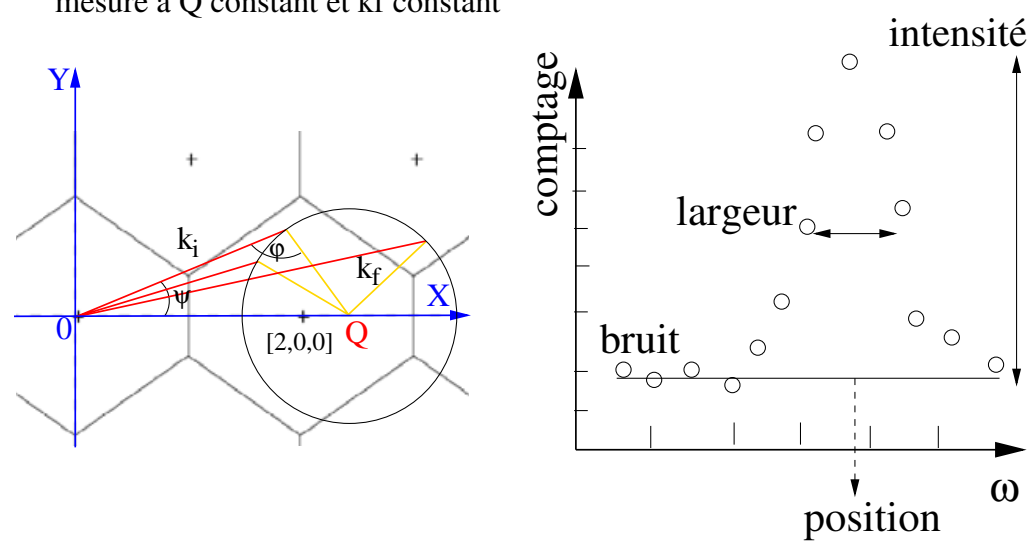

Figure 2. Principe de la mesure: on bouge les éléments du spectromètre pour parcourir pas à pas une trajectoire $\vec{Q}$ et $\omega$. Le comptage reporté permet de déterminer un point de la courbe de dispersion $\omega=f(\vec{q})$.

- source froide : Pour étendre le domaine d'investigation vers les basses énergies, on insère sur le trajet des neutrons un conteneur (bidon ou anneau) d'hydrogène ou deutérium liquide, où les neutrons perdent encore de l'énergie, ce qui recentre le flux autour de $\approx k_{i}=2 \AA^{-1}$ et permet des mesures dans une gamme de transfert typiquement de 0.015 à $3 \mathrm{THz}(0.05 \mathrm{meV}$ à $12 \mathrm{meV})$ (IN14 et IN12 à l'ILL, 4F1 et 4F2 au LLB.

- source chaude : Pour étendre le domaine jusqu'à $\approx 50 \mathrm{THz}(\approx 200 \mathrm{meV})$, on insère un bloc de graphite dont la température monte jusqu'à $2000^{\circ} \mathrm{C}$ sous l'effet du rayonnement $\gamma$ (IN1 à l'ILL).

Sur une source donnée, on a généralement le choix du monochromateur et de l'analyseur pour définir la qualité des faisceaux. La définition de $k_{i}$ et $k_{f}$ se faisant par la loi de Bragg $k=\pi /(d \sin \theta)$, la précision du faisceau, à collimation donnée, dépend de la distance interréticulaire $d$. On souhaite de plus des caractéristiques permettant :

- une bonne réflectivité, pour avoir un bon flux

- une mosaïcité raisonnable pour une bonne définition angulaire du faisceau

- la possibilité d'avoir des courbures horizontale et/ou verticale pour faire converger le faisceau

- éventuellement la possibilité de sélectionner le spin des neutrons

Les choix standards, pour des neutrons non polarisés, sont:

- le graphite pyrolytique PG[002] pour sa très bonne réflectivité,

- le Cuivre pour son choix de distance interréticulaire ([111], [2,0,0], [220]),

- le Germanium [111] ou Silicium [111] qui n'ont pas d'harmonique 2.

\subsection{Monochromateur}

Les caractéristiques des cristaux et la gamme de vecteur d'onde accessible en $k_{i}$ sont reportées dans le tableau suivant. Les valeurs minimum et maximum de $k_{i}$ sont calculées dans l'hypothèse d'un $2 \theta_{m}$ compris entre 16 et 75 degrés, pour un monochromateur unique.

Sur source thermique, PG[002] Ge[111] et Si[111] ont des distances interréticulaires voisines. PG[002] a une meilleur réflectivité, mais $\mathrm{Ge}[111]$ et $\mathrm{Si}[111]$ n'ont pas d'harmonique 2 (grâce à la structure diamant). Pour les mesures à grands transferts d'énergie, sur source thermique et $a$ 


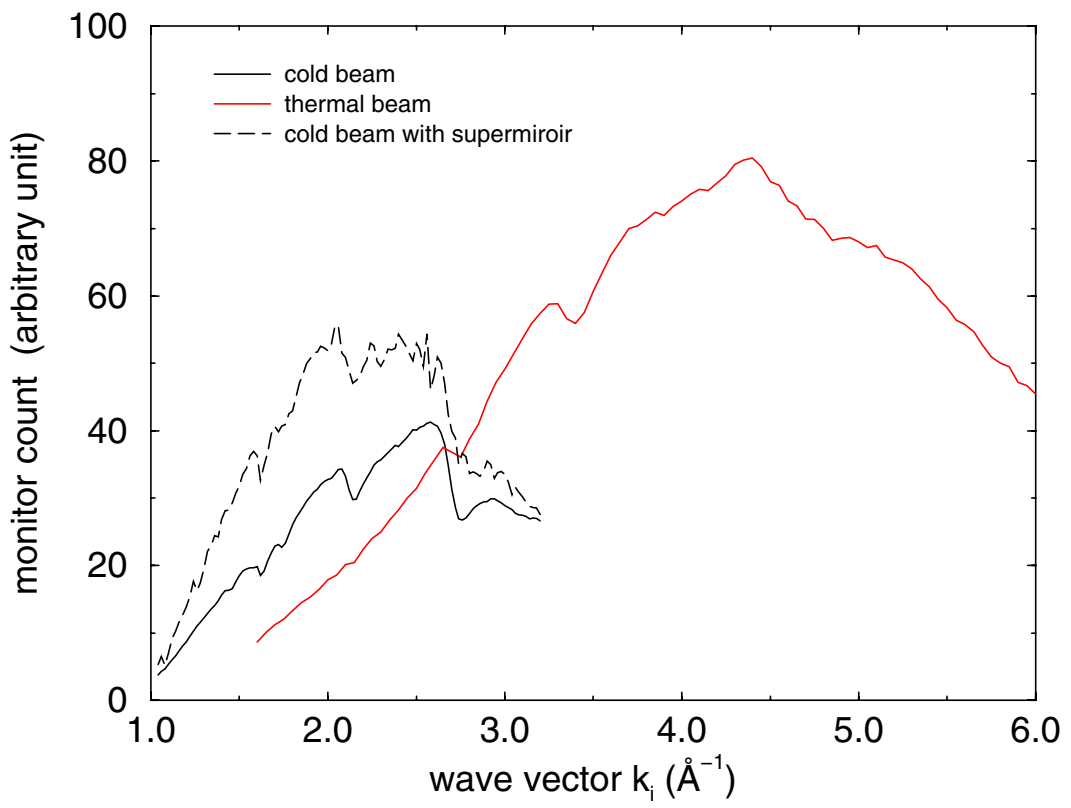

Figure 3. Exemples de distribution de flux de neutrons sur source froide (4F1 LLB) et source thermique (2T LLB). Sont reportées les valeurs du moniteur à temps constant, corrigées de la contamination par les harmoniques de $\mathrm{k}_{i}$.

\begin{tabular}{|l|c|c|}
\hline réflexion d $(\AA)$ & $k_{i} \min \left(\AA^{-1}\right)$ & $k_{i} \max \left(\AA^{-1}\right)$ \\
\hline PG [002] d=3.3535 & 1.54 & 6.73 \\
\hline $\mathrm{PG}[004] \mathrm{d}=1.6768$ & 3.08 & 13.46 \\
\hline $\mathrm{Si}[111] \mathrm{d}=3.1354$ & 1.65 & 7.2 \\
\hline $\mathrm{Cu}[111] \mathrm{d}=2.0869$ & 2.47 & 10.8 \\
\hline $\mathrm{Cu}[200] \mathrm{d}=1.8074$ & 2.86 & 12.5 \\
\hline $\mathrm{Cu}[220] \mathrm{d}=1.2780$ & 4 & 17.6 \\
\hline
\end{tabular}

fortiori sur source chaude $\mathrm{Cu}$ s'impose de [111] à [220] suivant la gamme et/ou la précision à atteindre.

Sur source froide, le PG[002] est le plus fréquent. Sur un canal du réacteur (4F1 et 4F2 au LLB), on peut utiliser un double monochromateur PG[002], car la réflectivité du PG[002] atteint 85 à $90 \%$ dans la gamme de $k_{i}$ utilisée. Cela permet d'atteindre des $k_{i}$ entre 1 et $3.4 \AA^{-1}$.

\subsection{Analyseur}

Sur toutes les sources, PG[002] est le plus fréquent. Si[111] peut être utilisé pour des propriétés de focalisation obtenues par une courbure mécanique, irréalisable avec PG[002].

\subsection{Neutrons polarisés}

l'alliage de Heusler est encore le meilleur candidat pour obtenir, sous champ magnétique, des faisceaux polarisés. Le filtre polarisant à $\mathrm{He}^{3}$ fait de gros progrès pour les neutrons chauds. Sur source froide, les "benders", guides courbés polarisants qui s'insèrent sur la trajectoire du faisceau, permettent de garder le PG[002]. 


\subsection{Remarque}

Une difficulté récurrente est liée au fait que la sélection par une réflexion de Bragg laisse passer des harmoniques $\left(2 \vec{k}_{i}, 3 \vec{k}_{i} \ldots\right)$. Nous verrons plus loin les possibilités de réduire les contaminations par les harmoniques.

\section{LES ÉLÉMENTS QUI INTERVIENNENT DANS LA MESURE, NOTIONS DE RÉSOLUTION}

En dehors de la complication lié à la maitrise de l'état de spin du neutron, le fait de travailler avec des neutrons polarisés n'intervient qu'au niveau de la définition de la section efficace de diffusion. Les considérations qui suivent s'appliquent donc à tout type de spectromètre 3-axes.

Après avoir défini les ordres de grandeur qui permettent de faire les choix de base: où trouver la source de neutrons équipée du monochromateur et de l'analyseur adaptés aux besoins d'une mesure donnée?, nous allons passer en revue les caractéristiques générales d'un spectromètre 3-axes et leur incidence sur la mesure : essentiellement l'intensité et la précision.

Cela pour préparer l'étape suivante: comment analyser les résultats de mesure pour en extraire proprement les informations recherchées.

L'appareil n'étant pas infiniment parfait, lorsqu'on fait une mesure en un point $\vec{Q}_{0}, \omega_{0}$, l'information obtenue correspond en fait à l'intégration sur un ensemble de points $\vec{Q}, \omega$ centré sur $\vec{Q}_{0}, \omega_{0}$. La fonction qui donne la pondération de cet ensemble est la fonction de résolution. L'intensité mesurée en un point $\vec{Q}_{0}, \omega_{0}$ est donnée par

$$
I\left(\vec{Q}_{0}, \omega_{0}\right)=\int S(\vec{Q}, \omega) R\left(\vec{Q}-\vec{Q}_{0}, \omega-\omega_{0}\right) \partial^{3} Q \partial \omega
$$

où $S(\vec{Q}, \omega)$ est la fonction de diffusion des neutrons. $R\left(\vec{Q}-\vec{Q}_{0}, \omega-\omega_{0}\right)$ est déterminée par l'ensemble des écarts à la mesure idéale. Avant une expérience, il est important de prévoir l'impact des conditions expérimentales sur la précision de la mesure et sur l'intensité espérée, ceci pour optimiser les conditions de mesure et analyser correctement les résultats obtenus.

Les caractéristiques à prendre en compte concernent toute la géométrie du spectromètre:

- taille des différents éléments: source, monochromateur, échantillon, analyseur, détecteur

- distances entre ces éléments

- définition angulaire du faisceau: collimations, diaphragmes, mosaïcité des cristaux, courbure horizontale et verticale du monochromateur et de l'analyseur.

Les corrélations entre ces éléments sont essentiellement liées à l'utilisation de monocristaux pour définir $\boldsymbol{k}_{i}$ et $\boldsymbol{k}_{f}$.

\subsection{Premier élément : réflexion par un monocristal}

Un monocristal est défini par sa distance interréticulaire $d_{0}=2 \pi / \tau_{0}$, par sa réflectivité $r_{0}$ et par sa distribution mosaïque, fonction de $\eta_{H}$ et $\eta_{V}$, horizontalement et verticalement. Un neutron sera réfléchi par ce monocristal s'il remplit la condition $\lambda_{0}=2 \pi / k_{0}=2 d_{0} \sin \left(\theta_{0}\right)$ qu'on peut écrire $2 \vec{k}_{0} \cdot \vec{\tau}_{0}=\tau_{0}^{2}$.

On définit le neutron par $\vec{k}\left(k_{0}+d k, \gamma, \delta\right)$, où $\gamma$ et $\delta$ sont les écarts angulaires, horizontal et vertical, à la trajectoire de référence et le monocristal (monochromateur ou analyseur) par $\vec{\tau}\left(\tau_{0}, \eta_{H}, \eta_{V}\right)$, soit en coordonnées cartésiennes:

$$
\vec{k}=\left\{\begin{array}{l}
\left(k_{0}+d k\right) \sin \left(\theta_{0}-\gamma\right) \cos (\delta) \\
\left(k_{0}+d k\right) \cos \left(\theta_{0}-\gamma\right) \cos (\delta) \\
\left(k_{0}+d k\right) \sin (\delta)
\end{array} \quad \vec{\tau}=\left\{\begin{array}{l}
\tau_{0} \cos \left(\eta_{H}\right) \cos \left(\eta_{V}\right) \\
\tau_{0} \sin \left(\eta_{H}\right) \cos \left(\eta_{V}\right) \\
\tau_{0} \sin \left(\eta_{V}\right)
\end{array}\right.\right.
$$


Le développement au premier ordre de la condition de réflexion entraîne les résultats exposés dans les paragraphes suivants :

\subsubsection{Composante dans le plan de diffusion :}

$d k \sin \left(\theta_{0}\right)-k_{0} \gamma \cos \left(\theta_{0}\right)+\eta_{H} k_{0} \cos \left(\theta_{0}\right)=0$ d'où $\eta_{H}=\frac{\gamma-d k}{k_{0}} \tan \left(\theta_{0}\right)$. Ceci veut dire que, si un monocristal est réglé pour réfléchir des neutrons de vecteur d'onde $\vec{k}_{0}$, les neutrons ayant des écart $d k$ et $\gamma$ en module et direction dans le plan de diffusion seront réfléchis avec une probabilité qui dépend de la distribution mosaïque du monochromateur.

En écrivant celle-ci, pour simplifier, $\exp -\eta_{H}^{2} / \eta_{0}^{2}$, cela donne :

$$
P_{H}\left(k_{0}+d k, \gamma\right)=\exp -\frac{\left(\gamma-\frac{d k}{k_{0}} \tan \left(\theta_{0}\right)\right)^{2}}{\eta_{0}^{2}}
$$

Après réflexion, le neutron fait avec la trajectoire idéale un écart angulaire horizontal $\gamma^{\prime}=\gamma-$ $2 \frac{d k}{k_{0}} \operatorname{tg}\left(\theta_{0}\right)$.

La mosä̈cité du monochromateur entraîne un élargissement dans la distribution des $k_{i}$ et $k_{f}$. Mais elle conditionne aussi la réflectivité du monochromateur, donc l'intensité du faisceau réfléchi.

\subsubsection{Composante perpendiculaire au plan de diffusion :}

La condition qui relie $\delta$ et $\delta^{\prime}$ avant et après l'échantillon est donnée par $\vec{k}-\vec{k}^{\prime}=\vec{\tau}$ qui entraîne $k_{0}(\delta-$ $\left.\delta^{\prime}\right)=\tau_{0} \eta_{V}$. Ceci signifie qu'un neutron incident ayant un écart angulaire vertical $\delta$ a la probabilité d'être diffusé suivant $\delta^{\prime}$ en fonction de la distribution de $\eta_{V}$, soit, dans une hypothèse gaussienne.

$$
1 / \sqrt{\pi} \eta_{0}^{\prime} \exp \left(-\eta_{V}^{2} / \eta_{0}^{\prime 2}\right)
$$

La mosaïcité verticale n'a qu'un effet de dispersion du faisceau réfléchi. L'idéal serait qu'elle soit nulle!

\subsection{Deuxième élément : courbure du monochromateur et de l'analyseur}

\subsubsection{La courbure horizontale}

Nous avons vu que la condition pour que le neutron soit réfléchi par une réflexion de Bragg d'un monocristal mosaïque s'écrivait $\eta_{H}=\gamma-\frac{d k}{k_{0}} \tan \left(\theta_{0}\right)$ et qu'après réflexion l'écart horizontal à la trajectoire était $\gamma^{\prime}=\gamma-2 \frac{d k}{k_{0}} \tan \left(\theta_{0}\right)$.

On a la même formulation si le monocristal est parfait mais présente une courbure horizontale, $\eta_{H}$ étant remplacé par l'angle $\alpha$ que fait le plan tangent à la courbure au point d'intersection.

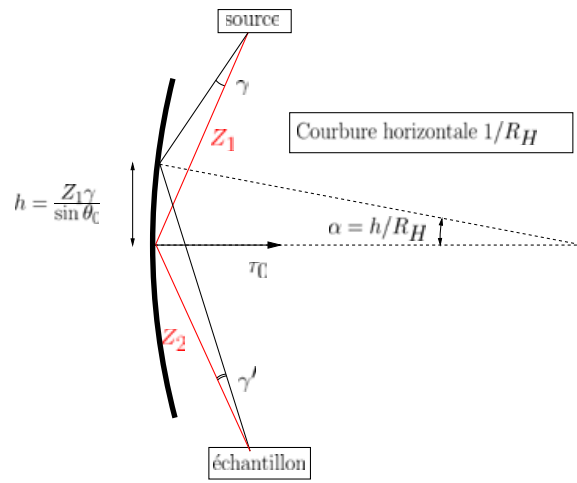

Si on suppose de plus que la source est ponctuelle, le neutron incident intercepte le cristal à une distance $h=$ $-Z_{1} \gamma / \sin \theta_{0}$ de son centre, si le rayon de courbure du monocristal est $R_{H}$, le plan réflecteur fait donc à cet endroit un angle $\alpha=-\frac{Z_{1} \gamma}{R_{H} \sin \theta_{0}}$ avec le plan de référence. La condition de réflexion impose $\alpha=\frac{d k}{k_{0}} \tan \theta_{0}-\gamma$ et pour focaliser le faisceau sur l'échantillon situé en $Z_{2}$, on doit réaliser la condition $Z_{1} \gamma=Z_{2} \gamma^{\prime}$. On en déduit $Z_{1} \gamma=$ $Z_{2} \gamma^{\prime}=Z_{2}\left(\gamma-2 \frac{d k}{k_{0}} \tan \theta_{0}\right)=Z_{2}(\gamma-2(\alpha-\gamma))$, soit $\left(Z_{1}+\right.$ $\left.Z_{2}\right) \gamma=-2 Z_{2} \alpha=2 \frac{Z_{1} Z_{2} \gamma}{R_{H} \sin \theta_{0}}$ qui donne la condition pour le rayon de courbure

$$
\frac{2}{R_{H} \sin \theta_{0}}=\frac{1}{Z_{1}}+\frac{1}{Z_{2}}
$$


La courbure horizontale est proportionnelle à $\sin \theta_{0}$, et donc inversement proportionnelle à $k_{0}$. Si on tient compte de la largeur de la source et de la mosaïcité horizontale du monochromateur, il n'y a pas de solution littérale, ni de focalisation parfaite. Mais la simulation permet de vérifier qu'on garde un effet de focalisation et d'obtenir une estimation raisonnable du gain. On en conclut que le bénéfice espéré est rapidement dégradé sous l'effet de la mosaïcité du monocristal lorsque les distances $Z_{1}$ ou $Z_{2}$ augmentent. La courbure horizontale est donc très efficace pour l'analyseur, où on peut réduire ces distances à 1 mètre et même moins. Par contre l'efficacité est réduite pour le monochromateur, sauf à utiliser un monochromateur avec une très faible mosaïcité (lame de Silicium courbée mécaniquement).

\subsubsection{La courbure verticale}

Nous avons déjà vu que la mosaïcité verticale n'avait qu'un effet négatif sur la distribution du faisceau. Heureusement, on peut quand même obtenir une focalisation verticale du faisceau.

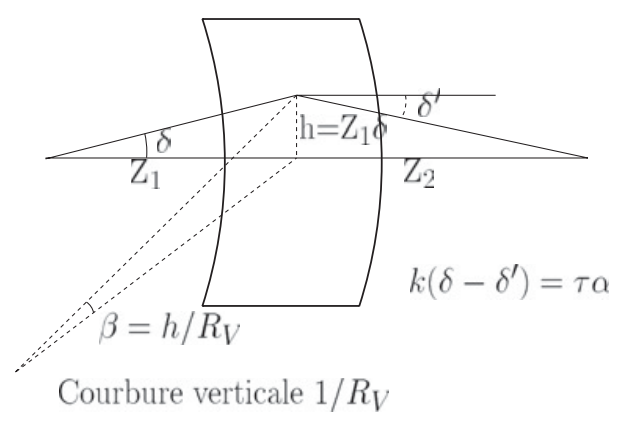

Raisonnons de nouveau sur une source ponctuelle et supposons la mosaïcité verticale nulle. Si le monocristal a une courbure verticale $\mathrm{R}_{V}$, l'angle $\beta$ au point d'incidence du neutron est $h / R_{V}$, avec $h=Z_{1} \delta$. La hauteur à la distance $\mathrm{Z}_{2}$ après la réflexion sera: $h^{\prime}=Z_{1} \delta+$ $Z_{2} \delta^{\prime}$. La condition de réflexion impose : $k_{0}\left(\delta-\delta^{\prime}\right)=$ $\tau_{0} \beta$, d'où: $h^{\prime}=Z_{2}\left(\delta^{\prime}+\frac{Z_{1}}{Z_{2}} \delta\right)=\delta\left(Z_{2}\left(1-\frac{\tau}{k_{0}} \frac{Z_{1}}{R_{V}}\right)+Z_{1}\right)$. On voit qu'on obtient $h^{\prime}=0$, quel que soit $\delta$, si on a:

$$
\frac{1}{Z_{1}}+\frac{1}{Z_{2}}=\frac{\tau}{k_{0}} \frac{1}{R_{V}}=\frac{2 \sin \theta_{0}}{R_{V}} .
$$

La courbure verticale est donc proportionnelle à $k_{0}$. La simulation permet de vérifier que la dégradation liée à la mosaïque verticale et à l'étalement de la source laissent malgré tout un gain appréciable, tant pour le monochromateur que pour l'analyseur.
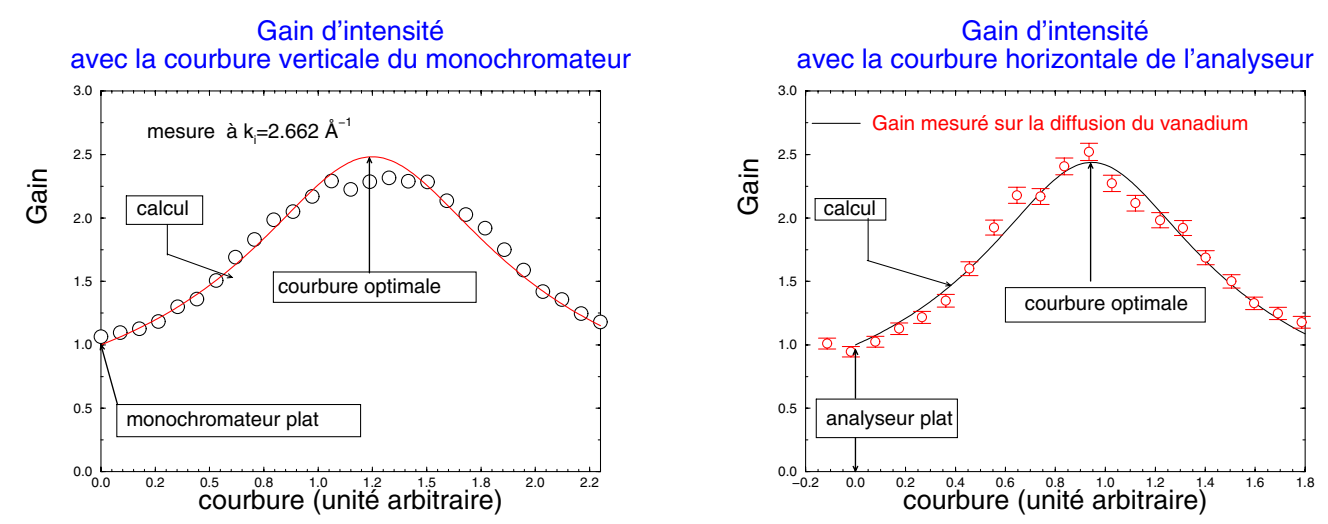

Figure 4. Gain de flux par courbure verticale du monochromateur (à gauche) et courbure horizontale de l'analyseur (à droite).

\subsubsection{Remarque}

Les gains obtenus en intensité grâce aux courbures du monochromateur et de l'analyseur ont des conséquences sur la qualité de la mesure! Mais le gain de flux obtenu grâce à ces courbures est particulièrement intéressant et est utilisé dans toutes les installations. 

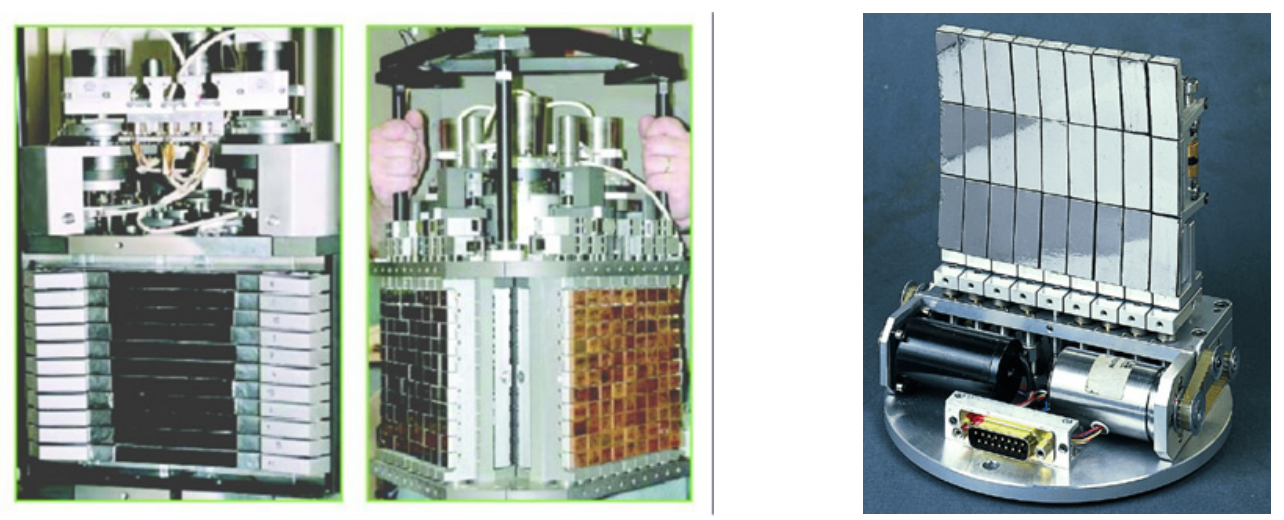

Figure 5. Exemples de monochromateur (à gauche) et d'analyseur (à droite) courbés.

\subsection{Troisième élément : divergences angulaires et collimateurs}

Les faisceaux divergent naturellement en raison de l'étendue de la source et de la mosaïque des cristaux. On peut réduire les divergences en plaçant sur la trajectoire du faisceau des collimateurs, assemblages de lames parallèles d'un matériau absorbant. Actuellement les lames sont généralement des feuilles plastiques comprenant du Gadolinium et maintenues tendues dans une structure métallique.

La probabilité de passage est alors donnée par une fonction triangle, qu'on peut assimiler à une gaussienne pour une description simplifiée de la fonction de résolution. La gamme utilisée varie de 10' à 60', largeur totale à mi-hauteur.

On utilise ces collimateurs essentiellement pour contrôler la divergence horizontale du faisceau, dans les cas où on a besoin d'une bonne précision en $\vec{Q}$. Il faut alors supprimer la courbure horizontale du monocristal impliqué, monochromateur ou analyseur. Ce choix entraîne une perte sensible d'intensité.

\subsection{Le comptage des neutrons: moniteur et détecteur}

\subsubsection{Le moniteur}

On a besoin de compter les neutrons avant l'échantillon pour faire la mesure à nombre de neutrons constant. Pour cela on utilise un moniteur. C'est un conteneur rempli d'un élément absorbant (généralement $\mathrm{He}^{3}$ ) avec un taux de remplissage qui fournit une efficacité d'absorption de quelques $10^{-6}$. Le prélèvement est sans incidence sur le flux envoyé sur l'échantillon et permet de ne pas saturer l'électronique de comptage.

L'efficacité du moniteur est inversement proportionnelle à $k_{i}$.

ATTENTION : L'utilisation d'une réflexion de Bragg pour la sélection de $k_{i}$ entraîne l'existence des harmoniques $\mathrm{n} k_{i}$. Si on ne peut pas filtrer ces harmoniques, il faut appliquer une correction à la valeur du moniteur pour déterminer le nombre de neutrons correspondant à $k_{i}$ seul.

\subsubsection{Le détecteur}

L'élément final de la chaîne de mesure est le détecteur. C'est un cylindre rempli d'un mélange de gaz, dont $\mathrm{He}^{3}$ sous pression pour obtenir une efficacité de $100 \%$ pour tout $k_{f}$. Une anode sous haute tension transforme l'absorption d'un neutron par $\mathrm{He}^{3}$ en impulsion électrique, calibrée et transmise à une électronique de détection. $\mathrm{He}^{3}$ étant aussi sensible aux rayons $\gamma$, une discrimination électronique est utilisée pour sélectionner les signaux propres aux neutrons. 
Le détecteur est au centre d'une protection destinée à minimiser le bruit de fond. L'extérieur de cette protection est souvent en polyéthylène, afin de ralentir les éventuels neutrons rapides. Le noyau central est fait d'un absorbant suffisamment épais pour arrêter la quasi-totalité des neutrons ne suivant pas le chemin prévu pour $\vec{k}_{f}$.

\subsection{Le bruit de fond : qualité de la mesure et radioprotection}

Les sections efficaces de diffusion inélastique des neutrons sont faibles et les sources d'une intensité modeste comparée aux sources de rayons X. Il est donc important d'éliminer ou tout au moins de minimiser tout signal parasite qui pourrait affecter la mesure.

On ne peut pas grand chose contre la diffusion inélastique incohérente de l'échantillon, sauf dans quelques cas où le choix d'un isotope précis peut avoir une grande incidence. La substitution de $\mathrm{H}$ par $\mathrm{D}$ est ainsi quasiment impérative pour une mesure propre.

Par contre, il faut rigoureusement contrôler tout ce qu'on met dans le faisceau de neutrons et qui pourrait devenir une source de bruit de fond: support échantillon, colle, conteneur. Il faut masquer tout ce qui peut l'être et définir, par des diaphragmes, le faisceau à sa taille strictement utile, compte tenu de la dimension de l'échantillon.

ATTENTION : La capture d'un neutron entraîne de façon automatique l'émission d'autres particules, généralement des rayons $\gamma$.

Il ne faut pas oublier la sécurité des personnes! On choisit donc les absorbants en fonction du flux mis en jeu et de l'endroit où on souhaite intervenir.

Les protections standard du spectromètre sont :

- La protection des monochromateurs.

Elle reçoit le faisceau primaire polychromatique mais doit arrêter tous les neutrons autres que le faisceau monochromatique utilisé pour la mesure. Elle est réalísée en béton de haute densité et certifiée par le service de radio-protection. Un obturateur primaire permet d'ouvrir ou fermer le faisceau primaire polychromatique. Un obturateur secondaire permet de fermer le faisceau monochromatique.

Dans la majorité des cas, une fois le faisceau fermé, l'échantillon est la principale source de rayonnement. Renseignez-vous sur son degré d'activation à court et long terme!

- La protection du faisceau non diffusé par l'échantillon.

Un "beam-stop" est en place sur la trajectoire du faisceau incident, le plus près possible après l'échantillon. L'absorbant est généralement à base de $\mathrm{B}_{4} \mathrm{C}$ en sandwich dans du plomb.

- La protection du faisceau diffusé vers l'analyseur.

Les éléments de protection sur le trajet des "bons" neutrons sont aussi à base de $\mathrm{B}_{4} \mathrm{C}$. Ils peuvent être rigides si le $\mathrm{B}_{4} \mathrm{C}$ est mélangé avec de l'araldite ou souples s'il est mélangé avec un epoxy ad hoc. La charge en hydrogène permet de ralentir les neutrons et de faciliter leur capture par le $\mathrm{B}_{4} \mathrm{C}$. L'intérieur de ces protections est tapissé d'une feuille de Cadmium pour éviter la rétrodiffusion par l'hydrogène sur la surface.

- La protection du faisceau diffusé par l'échantillon et son environnement.

L'échantillon est susceptible de diffuser dans $4 \pi$ stéradian, de façon cohérente et incohérente. Il est important d'essayer de fermer au maximum l'angle solide en dehors de la trajectoire conduisant à l'analyseur.

Le Cadmium et le Gadolinium sont d'excellents absorbants, mais les $\gamma$ de capture qu'ils émettent sont dangereux, ce qui restreint leur usage. Des diaphragmes télécommandables permettent de réduire les dimensions du faisceau pour l'ajuster aux dimensions utiles, fonction de l'échantillon. 


\section{RÉALISATION D'UNE MESURE}

L'objectif de la mesure permet des prévisions sur le domaine d'énergie et l'ordre de grandeur de la précison requise. Cela suffit en général pour définir la source de neutrons la mieux adaptée. Il reste à définir les paramètres ajustables d'un spectromètre. Pour cela, il faut d'abord prendre en compte le domaine $(\vec{Q}, \omega)$ pertinent pour la physique visée.

\subsection{Domaine $\overrightarrow{\boldsymbol{Q}}, \omega$ et précision de la mesure}

Pour une mesure de phonons, le poids en $Q^{2}$, bien qu'un peu atténué par le facteur Debye-Waller, favorise les grandes valeurs de $\vec{Q}$. Mais si on veut suivre une transition de phase, on veut aussi atteindre les basses énergies avec une bonne précision.

Pour une étude magnétique, on sait que l'intensité va décroitre comme le carré du facteur de forme magnétique, ce qui favorise les petites valeurs de $\vec{Q}$. Mais si on a besoin de mesurer à haute énergie, il faut qu' on puisse fermer le triangle $\vec{k}_{i}, \vec{k}_{f}, \vec{Q} \ldots$

Dans tous les cas une valeur $(\vec{Q}, \omega)$ peut être obtenue de diverses façons, en fonction du choix de $\mathrm{k}_{i}$ ou $\mathrm{k}_{f}$. Il faut donc évaluer les avantages et les inconvénients des choix possibles. L'élément décisif est souvent la précision nécessaire pour obtenir l'information recherchée.

La figure 6 donne quelques exemples de domaines accessibles sur source froide et thermique, pour différents monochromateurs. On voit que le domaine accessible est vaste, mais avec une forte corrélation entre $Q$ et $\omega$. La figure 7 illustre la dépendance en fréquence de la précision de la mesure pour une mesure à $Q$ constant d'un mode non dispersif. Cette précision dépend du choix du monochromateur et de l'analyseur et du choix de $k_{i}$ ou $k_{f}$ constant.
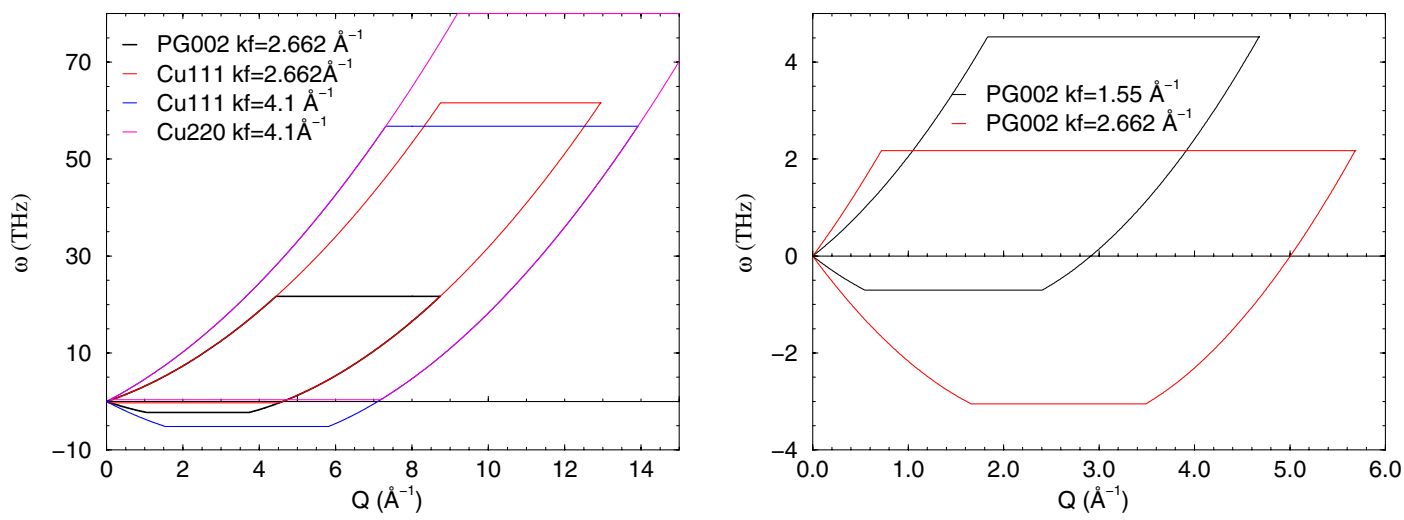

Figure 6. Exemples de domaines $(\vec{Q}, \omega)$ accessibles sur une source thermique (2T LLB à gauche) ou froide (4F1 et 4F2 LLB à droite), compte tenu des limites angulaires du spectromètre et du choix du monochromateur.

\section{$5.2 k_{i}$ ou $k_{f}$ constant, filtrage des harmoniques}

A priori rien n'empèche de faire une mesure où $k_{i}$ et $k_{f}$ varieraient pas à pas. En pratique on n'utilise presque exclusivement la mesure à $k_{f}$ constant. La raison essentielle est issue d'une étude de la fonction de résolution de l'appareil. En effet la mesure du flux incident par un moniteur, d'efficacité inversement proportionnelle à $k_{i}$, permet une prise en compte automatique des corrections liées à la distribution du flux incident, évite les corrections de réflectivité de l'analyseur, et s'accorde mieux à la gestion des problèmes liés à la présence des harmoniques de $k_{i}$ et $k_{f}$. 


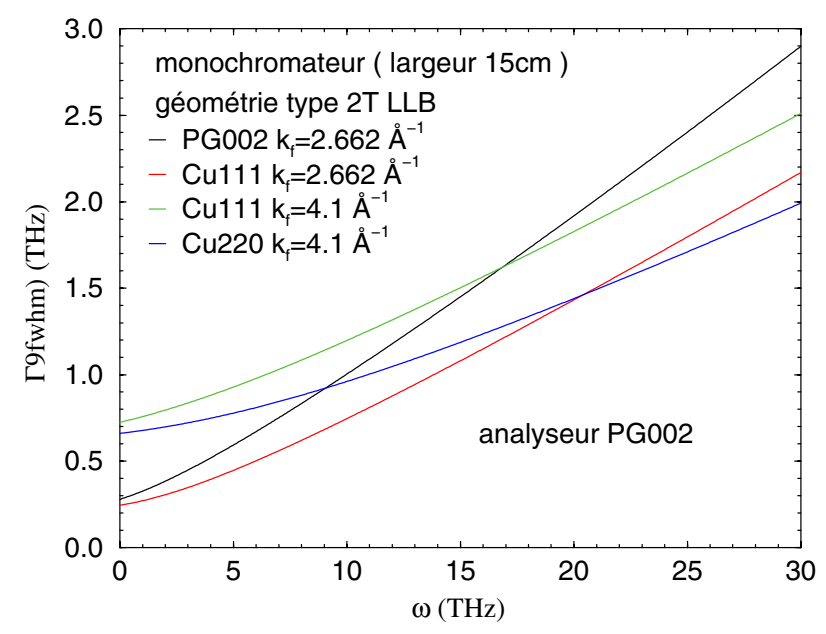

Figure 7. Exemples de variation de la précision en énergie, pour différentes conditions de mesure. La largeur totale à mi-hauteur (fwhm) est estimée pour un mode non dispersif.

\subsubsection{Le filtrage des harmoniques}

Sauf exceptions liées à la structure cristalline du monochromateur et de l'analyseur, le faisceau incident contient les harmoniques $m k_{i}$ et l'analyseur laisse passer les harmoniques $n k_{f}$. Pour une position où on souhaite mesurer $\vec{k}_{i}-\vec{k}_{f}=\vec{Q}$ et $k_{i}^{2}-k_{f}^{2}=\alpha \nu$, on a la possibilité de mesurer $m \vec{k}_{i}-n \vec{k}_{f}=\vec{Q}^{\prime}$ et $m^{2} k_{i}^{2}-n^{2} k_{f}^{2}=\alpha v^{\prime}$. Cela fait beaucoup...

La méthode de filtrage la plus utilisée profite d'anomalies de transmission de composés qu'on peut interposer sur le faisceau.

- Dans le domaine des neutrons chauds on utilise des absorptions résonnantes pour éliminer l'harmonique 2.

- Sur les spectromètres 3-axes on préfère généralement travailler à $k_{f}$ constant et utiliser un filtre en graphite pour réduire les harmoniques. Le filtre est un bloc de graphite d'environ $5 \mathrm{~cm}$ d'épaisseur, empilement de plan graphite suivant l'axe [001], avec une mosaïcité d'environ 3 degrés. Les anomalies de transmission sont liées à la diffusion du faisceau par des raies de type poudre du plan. Pour la valeur de $2.662 \AA^{-1}$, la transmission de l'harmonique 2 est de l'orde de $3.10^{-3}$, à condition que l'axe [001] du graphite soit bien aligné sur l'axe du faisceau! C'est ce qui explique l'utilisation dominante de cette valeur. Sur les sources froides, où la population des harmoniques de $k_{i}$ est plus faible, on utilise aussi les valeurs $k_{f}=1.97$ ou $1.64 \AA^{-1}$, souvent en doublant l'épaisseur du filtre.

- Sur les spectromètres 3-axes sur source froide on utilise du béryllium sous forme frittée. Si on interpose un bloc d'une vingtaine de centimètres sur le faisceau, la transmission des neutrons de $k>1.59 \AA^{-1}$ tombe à quelques $10^{-4}$ et reste proche de $80 \%$ pour les $k$ inférieurs, si on refroidit le béryllium à la température de l'azote liquide, pour réduire la diffusion par les phonons. On travaille souvent avec le filtre béryllium sur $k_{f}$ pour n'avoir aucune harmonique sur l'analyseur. Mais il ne faut pas oublier l'existence d'harmoniques sur $k_{i} \ldots$

- On peut aussi, en principe, utiliser un filtrage mécanique du faisceau à l'aide d'un sélecteur de vitesse. Les problèmes mécaniques que posent ces sélecteurs sont peu compatibles avec la flexibilité du spectromètre 3-axes et entraînent un coût de fabrication et une maintenance, jusqu'ici prohibitifs... 


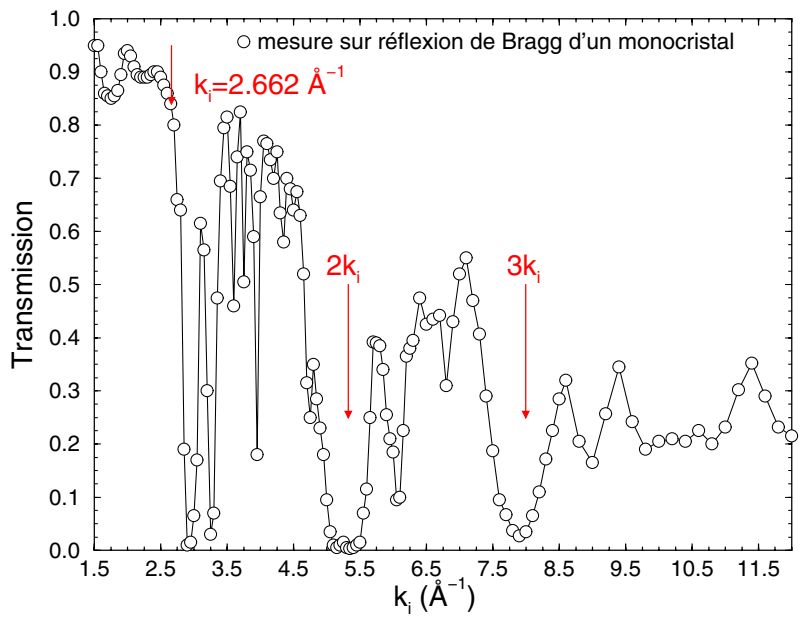

Figure 8. La transmission d'un filtre graphite. Les anomalies de transmission explique pourquoi la valeur $k_{f}=$ $2.662 \AA^{-1}$ est si souvent utilisée.

\subsubsection{Mesure à $\omega$ ou $\vec{Q}$ constant}

De même qu'il n'y a pas d'obligation stricte de $k_{i}$ ou $k_{f}$ constant, rien n'oblige à réaliser une mesure à $\vec{Q}$ ou $\omega$ constant. Les arguments qui conduisent à ce choix sont :

- liés à la physique: la plupart des quantités d'intérêt qu'on cherche à atteindre sur un monocristal, dispersion, amortissement, facteur de structure, sont fonction de $\vec{q}$, vecteur dans la première zone de Brillouin.

- liés à la résolution de l'appareil: Si un mode présente une forte dispersion, la mesure sera plus propre à $\omega$ constant. Mais c'est généralement au détriment de la détermination d'un amortissement.

\section{EFFETS LIÉS À LA RÉSOLUTION DU SPECTROMÈTRE}

Nous sommes maintenant prêts à faire des choix pour réaliser une mesure adaptée à la physique de l'échantillon étudié. Il reste que la taille de l'échantillon ou la section efficace du phénomène étudié peuvent amener à un compromis pour obtenir une intensité raisonnable dans le temps de faisceau imparti. Il est donc important de connaître quelques effets systématiques liés à la fonction de résolution du spectromètre 3-axes, afin de ne pas être tenté de voir des effets révolutionnaires là où il n'y a qu'un effet d'appareillage.

La fonction de résolution du spectromètre est en première approximation décrite comme une distribution gaussienne centrée au point $\vec{Q}_{0}, \omega_{0}$ de la mesure, dont les surfaces à niveau constant sont des ellipsoïdes dans un espace à 4 dimensions. Les exemples suivants illustrent les effets liés à la convolution de cette distribution avec la section efficace de diffusion inélastique des neutrons d'une excitation de type phonon ou magnon, sans amortissement.

\subsection{Mesure à énergie constante d'un mode dispersif - focalisation}

Comme illustré sur la figure 9, à section efficace constante l'intensité mesurée dépend beaucoup de la géométrie de la mesure. En configuration transverse, on observe un effet de convolution, plus ou moins prononcé suivant la valeur de la dispersion. Cet effet est systématique et le côté focalisé dépend de la 

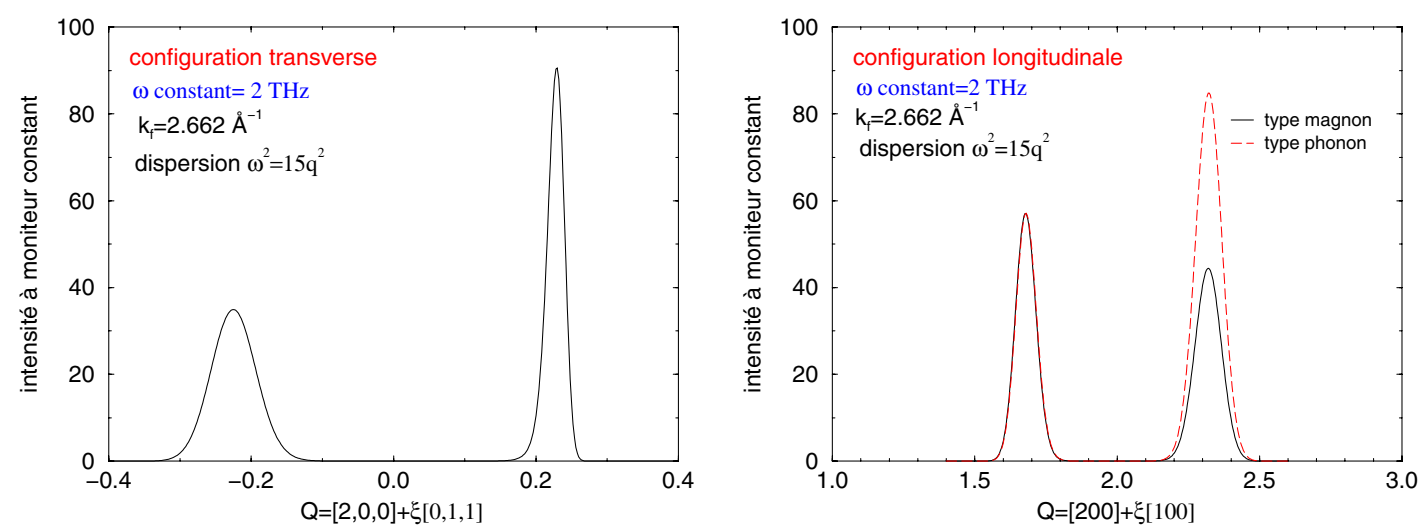

Figure 9. La convolution de la fonction de résolution avec la nappe de dispersion entraîne un effet de focalisation en configuration transverse. Pour un phonon le poids en $Q^{2}$ est à prendre en compte en configuration longitudinale.
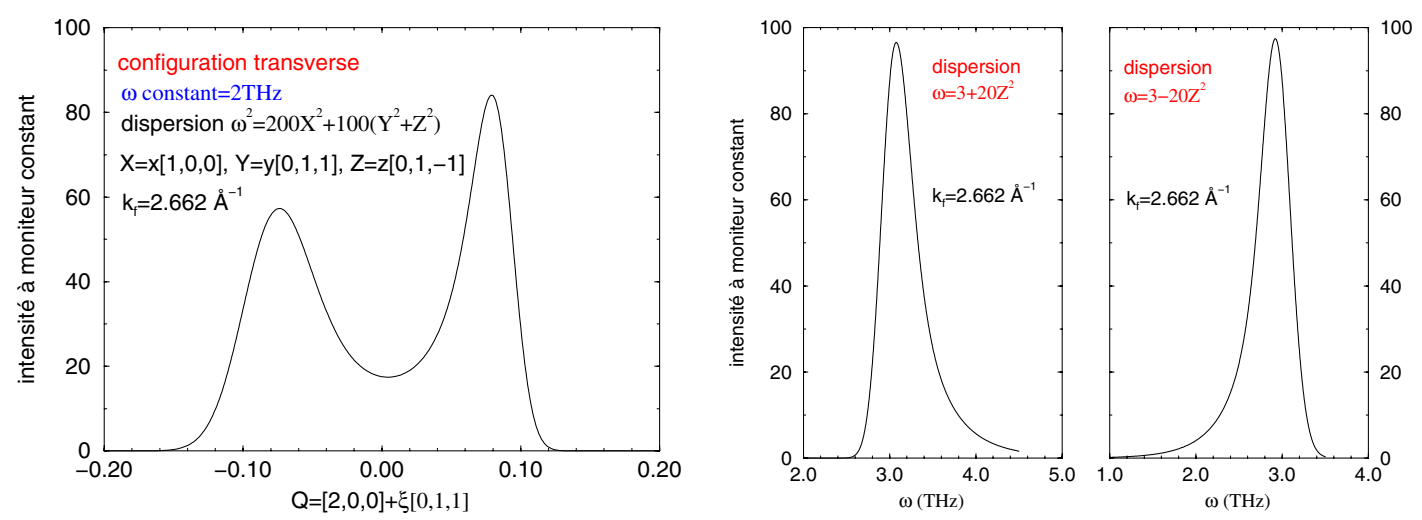

Figure 10. -à gauche: Quand la dispersion devient forte et/ou est asymétrique, le profil de la mesure devient difficile à analyser -à droite une asymétrie de la mesure peut être dûe à la dispersion perpendiculairement au plan de diffusion.

géométrie du spectromètre (faites-le vous préciser pour le spectromètre où vous faites la mesure!). Sur le pic focalisé en configuration transverse, on peut remarquer un léger écart à la forme gaussienne. Ceci résulte de la courbure de la nappe de dispersion. Ces effets de courbure sont encore plus prononcés quand la dispersion est forte et asymétrique. Ainsi une mesure ayant un profil similaire à celui reporté à gauche dans la figure 10 ne peut plus être interprétée simplement. La simulation a été réalisée avec un mode sans amortissement, mais des effets d'amortissement ou d'asymétrie de dispersion pourrait conduire à des profils similaires.

On peut également constater que si on utilise la valeur de $\xi$ au maximum d'intensité pour déduire la pente, soit $\xi \approx 0.079$, sachant que le paramètre de maille vaut $4 \AA$, on obtient une pente largement différente de la pente réelle: $11.4 \mathrm{THz} . \AA$ au lieu de 10 ! C'est aussi un effet lié à la convolution avec la fonction de résolution. Il est important d'en tenir compte si on veut déduire de la mesure des vitesses du son par exemple.

\subsection{Mesure à $\vec{Q}$ constant}

Si le mode n'est pas trop dispersif, on a intérêt à réaliser la mesure à $\vec{Q}$ constant. Les paramètres physiques - position, amortissement et facteur de structure - ont généralement une dépendance en $\vec{Q}$ 
modérée et la mesure donne alors des valeurs qu'on peut assimiler à celles de la valeur moyenne de $|\vec{Q}|$. Il faut se rappeler que l'on utilise une divergence angulaire verticale largement supérieure à la divergence horizontale. La valeur moyenne $q^{2}=q^{2}$ (in-plane) $+q_{\perp}^{2}$ est d'autant plus affectée par $q_{\perp}^{2}$ que $q$ est petit, donc près du centre de zone.

Cet effet de divergence verticale se remarque aussi lorsque la courbe de dispersion mesurée dans le plan de diffusion est un extremum de la nappe de dispersion (fond de vallée ou crête), comme illustré à droite dans la figure 10.

Lorsqu'on a une dispersion suivant localement une loi $\omega=\omega_{0}+D\left(\vec{q}-\vec{q}_{0}\right)^{2}$ (onde de spin ferromagnétique par exemple) ou $\omega^{2}=\omega_{0}^{2}+C^{2}\left(\vec{q}-\vec{q}_{0}\right)^{2}$ (phonon amorti au voisinage d'un point de transition ou onde de spin antiferromagétique), la détermination du gap $\omega_{0}$ n'est pas aussi évidente qu'on le souhaiterait. . .
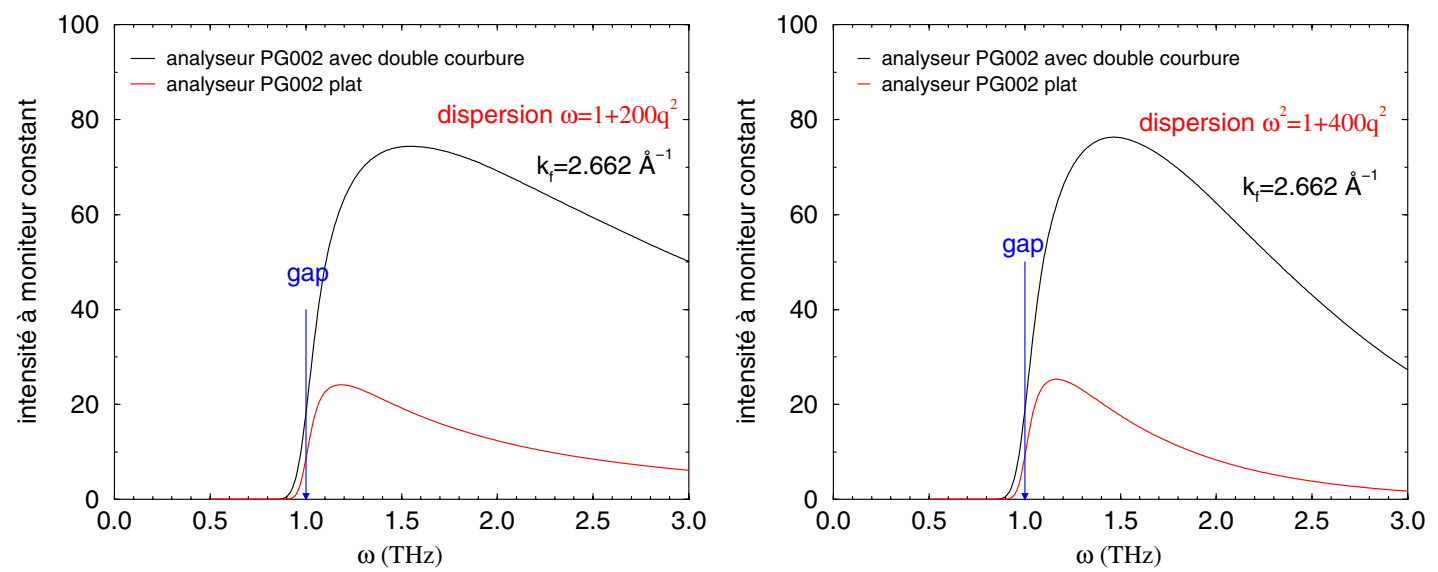

Figure 11. À gauche : exemple d'onde de spin ferromagnétique. À droite : exemple d'onde de spin antiferromagnétique. Utiliser l'analyseur courbé augmente largement l'intensité mesurée, mais ne permet pas une bonne détermination du gap.

\section{ARTEFACTS (SPURIONS)}

\section{1 les combinaisons d'harmoniques de $\boldsymbol{k}_{\boldsymbol{i}}$ ou $\boldsymbol{k}_{\boldsymbol{f}}$}

Nous avons déjà évoqué les difficultés liées à la présence des harmoniques sur $k_{i}$ ou $k_{f}$. Elles ont au moins le mérite d'être prévisibles...Pour une mesure à énergie variable et $k_{f}$ constant, le spectromètre est en position pour obtenir $k_{i}^{2}-k_{f}^{2}=\alpha \omega(\alpha=1.9959$ si $\omega$ est en THz). Les combinaisons d'harmoniques donnent les schémas définis par $m^{2} k_{i}^{2}-n^{2} k_{f}^{2}=\alpha \omega^{\prime}$, où $m$ et $n$ sont des entiers autorisés par les structures cristallines du monochromateur et de l'analyseur.

Le risque dominant correspond aux valeurs pour lesquelles $\omega^{\prime}$ est nul, qui permettent la détection de processus élastiques cohérents ou incohérents, provenant de l'échantillon ou de son environnement. On en déduit les positions à risque: $\alpha \omega=k_{f}^{2}\left(n^{2} / m^{2}-1\right)$. Pour une mesure réalisée à $k_{f}=2.662 \AA^{-1}$ ou $4.1 \AA^{-1}$ les valeurs à risque sont donc :

\begin{tabular}{|c|c|c|c|c|c|}
\hline \multicolumn{2}{|c|}{} & \multicolumn{2}{c|}{$k_{f} 2.662 \AA^{-1}$} & \multicolumn{2}{c|}{$k_{f} 4.1 \AA^{-1}$} \\
\hline $\mathrm{m}$ & $\mathrm{n}$ & $\omega \mathrm{THz}$ & $\omega \mathrm{meV}$ & $\omega \mathrm{THz}$ & $\omega \mathrm{meV}$ \\
\hline 1 & 2 & 10.65 & 44.04 & 25.26 & 104.47 \\
\hline 2 & 3 & 4.44 & 18.36 & 10.53 & 43.55 \\
\hline 3 & 4 & 2.76 & 11.42 & 6.55 & 27.0 \\
\hline
\end{tabular}


Il ne faut pas oublier la combinaison élémentaire : $2 \vec{k}_{i}, 2 \vec{k}_{f} \Longrightarrow 2 \vec{Q}, 4 \omega$, surtout si on se contente d'un filtrage modeste avec $k_{f}=1.97$ ou $1.64 \AA^{-1} \ldots$

\subsection{Les diffusions parasites}

Le faisceau incident ne contient pas que des neutrons de vecteur d'onde $k_{i}$ ou de ses harmoniques. Il est accompagné d'un faisceau blanc qui provient de toutes les diffusions possibles autres que celle associée à la réflexion de Bragg sur le monochromateur. On y retrouve en particulier une population de neutrons de vecteur d'onde $k_{f}$. Si la configuration de l'échantillon permet la diffusion par un pic de Bragg pour la valeur $k_{f}$, ou si l'angle de diffusion correspond à celui, pour $k_{f}$, d'une raie de poudre d'un matériau présent dans le faisceau au voisinage de l'échantillon (aluminium particulièrement!), on aura un pic d'intensité dans la mesure. Ce type de signal parasite ne peut être démontrer qu'en refaisant (à temps constant) la mesure concernée après avoir décalé le monochromateur!

Il ne faut pas non plus oublier que le faisceau incident peut être diffusé sous forme de raie de poudre, ou de raie de Bragg de l'échantillon, pour la valeur $k_{i}$. Si ces diffusions sont intenses, le faisceau parasite qu'elles forment peut être diffusé de façon incohérente par l'analyseur et son environnement et créer ainsi un pic d'intensité parasite dans la mesure.

Ce type de parasite peut être détecté par l'interposition, sur le trajet échantillon-analyseur, d'un compteur de type moniteur, à efficacité faible pour ne pas réduire significativement le bon signal.

\section{FORMALISATION DE LA RÉSOLUTION}

Il ne suffit pas de mesurer, il faut extraire de la mesure les quantités pertinentes... Nous avons posé les prémices de ce problème délicat en indiquant les conditions de franchissement du monochromateur et de l'analyseur et les possibilités de courbure. Pour progresser dans la maîtrise de ce sujet, on peut repartir de l'approche initiale simplifiée de Cooper et Nathans ref [1], normalisée par B. Dorner ref [2] où on ne gardeque la probabilité de franchir des monocristaux plats, ayant une mosaïcité de type gaussien, et des collimateurs à lames, dont la probabilité de passage sera assimilée à une gaussienne.

\subsection{L'étape Cooper-Nathans}

On note les écarts angulaires $\gamma$ dans le plan de diffusion et $\delta$ suivant la perpendiculaire à ce plan. Suivant Cooper et Nathans nous utiliserons les indices $0,1,2,3$ pour les segments source-monochromateur, monochromateur-échantillon, échantillon-analyseur et analyseur-détecteur. Le neutron incident est défini par $k_{i}, d k, \gamma_{1}, \delta_{1}$ et le neutron diffusé par $k_{f}, d k^{\prime}, \gamma_{2}, \delta_{2}$.

Le passage du monochromateur impose $\gamma_{0}=\gamma_{1}+2 \frac{d k}{k_{i}} \operatorname{tg}\left(\theta_{m}\right)$ et celui de l'analyseur $\gamma_{3}=\gamma_{2}-$ $2 \frac{d k^{\prime}}{k_{f}} \operatorname{tg}\left(\theta_{a}\right) . r_{m}$ et $r_{a}$ sont les réflectivités du monochromateur et de l'analyseur. La probabilité de passage du neutron est donc :

$$
\begin{aligned}
& \mathcal{P}_{\mathrm{H}_{i}} \text { avant échantillon } \quad r_{m} \exp -\frac{\left(\gamma_{1}+2 \frac{d k}{k_{i}} \tan \theta_{m}\right)^{2}}{\alpha_{0}^{2}} \exp -\frac{\left(\gamma_{1}+\frac{d k}{k_{i}} \tan \theta_{m}\right)^{2}}{\eta_{m}^{2}} \exp -\frac{\gamma_{1}^{2}}{\alpha_{1}^{2}} \\
& \mathcal{P}_{\mathrm{V}_{i}} \text { avant échantillon } * \frac{\beta_{0}}{\sqrt{\beta_{0}^{2}+4 \eta_{m}^{\prime 2} \sin ^{2} \theta_{m}}} \exp -\left(\frac{\delta_{1}^{2}}{\beta_{0}^{2}+4 \eta_{m}^{\prime 2} \sin ^{2} \theta_{m}}\right) \exp -\frac{\delta_{1}^{2}}{\beta_{1}^{\prime 2}} \\
& \mathcal{P}_{\mathrm{H}_{f}} \text { après échantillon } r_{a} \exp -\frac{\gamma_{2}^{2}}{\alpha_{2}^{2}} \exp -\frac{\left(\gamma_{2}-\frac{d k^{\prime}}{k_{f}} \tan \theta_{a}\right)^{2}}{\eta_{a}^{2}} \exp -\frac{\left(\gamma_{2}-2 \frac{d k^{\prime}}{k_{f}} \tan \theta_{a}\right)^{2}}{\alpha_{3}^{2}} \\
& \mathcal{P}_{\mathrm{V}_{f}} \text { après échantillon } * \frac{\beta_{3}}{\sqrt{\beta_{3}^{2}+4 \eta_{a}^{\prime 2} \sin ^{2} \theta_{a}}} \exp -\left(\frac{\delta_{2}^{2}}{\beta_{3}^{2}+4 \eta_{a}^{\prime 2} \sin ^{2} \theta_{a}}\right) \exp -\frac{\delta_{2}^{2}}{\beta_{2}^{\prime 2}}
\end{aligned}
$$


on obtient :

$$
\begin{aligned}
\mathcal{P}_{H} & =P_{H_{i}} \times P_{H_{f}} \\
& =r_{m} r_{a} \exp -\left(A_{1} d k^{2}+2 B_{1} d k \gamma_{1}+C_{1} \gamma_{1}^{2}\right) \exp -\left(A_{2} d k^{\prime 2}+2 B_{2} d k^{\prime} \gamma_{2}+C_{2} \gamma_{2}^{2}\right)
\end{aligned}
$$

et

$$
\begin{aligned}
\mathcal{P}_{V} & =P_{V_{i}} \times P_{V_{f}} \\
& =\frac{\beta_{0}}{\sqrt{\beta_{0}^{2}+4 \eta_{m}^{\prime 2} \sin ^{2} \theta_{m}}} \frac{\beta_{3}}{\sqrt{\beta_{3}^{2}+4 \eta_{a}^{\prime 2} \sin ^{2} \theta_{a}}} \exp -D_{1} \delta_{1}^{2} \exp -D_{2} \delta_{2}^{2}
\end{aligned}
$$

avec:

$$
\left\{\begin{array} { l } 
{ A _ { 1 } = \frac { \operatorname { t a n } ^ { 2 } \theta _ { m } } { k _ { i } ^ { 2 } } ( \frac { 4 } { \alpha _ { 0 } ^ { 2 } } + \frac { 1 } { \eta _ { m } ^ { 2 } } ) } \\
{ B _ { 1 } = \frac { \operatorname { t a n } \theta _ { m } } { k _ { i } } ( \frac { 2 } { \alpha _ { 0 } ^ { 2 } } + \frac { 1 } { \eta _ { m } ^ { 2 } } ) } \\
{ C _ { 1 } = \frac { 1 } { \alpha _ { 0 } ^ { 2 } } + \frac { 1 } { \eta _ { m } ^ { 2 } } + \frac { 1 } { \alpha _ { 1 } ^ { 2 } } } \\
{ D _ { 1 } = \frac { 1 } { \beta _ { 1 } ^ { 2 } } + \frac { 1 } { \beta _ { 0 } ^ { 2 } + 4 \eta _ { m } ^ { \prime 2 } \operatorname { s i n } ^ { 2 } \theta _ { m } } }
\end{array} \left\{\begin{array}{l}
A_{2}=\frac{\tan ^{2} \theta_{a}}{k_{f}^{2}}\left(\frac{4}{\alpha_{3}^{2}}+\frac{1}{\eta_{a}^{2}}\right) \\
B_{2}=-\frac{\tan \theta_{a}}{k_{f}}\left(\frac{2}{\alpha_{3}^{2}}+\frac{1}{\eta_{a}^{2}}\right) \\
C_{2}=\frac{1}{\alpha_{2}^{2}}+\frac{1}{\eta_{a}^{2}}+\frac{1}{\alpha_{3}^{2}} \\
D_{2}=\frac{1}{\beta_{2}^{2}}+\frac{1}{\beta_{3}^{2}+4 \eta_{a}^{\prime 2} \sin ^{2} \theta_{a}}
\end{array}\right.\right.
$$

8.1.1 Nombre de neutrons incidents. Intégrale de la résolution

Le nombre de neutrons incidents est proportionnel à l'intégrale $V_{i}=\int P_{H_{i}} P_{V_{i}} k_{i}^{2} \partial d k \partial \gamma_{1} \partial \delta_{1}$, soit $V_{i}=$ $\frac{\pi k_{i}^{2}}{\eta_{m} \sqrt{\left(A_{1} C_{1}-B_{1}^{2}\right) D 1}} \frac{\beta_{0}}{\sqrt{\beta_{0}^{2}+4 \eta_{m}^{\prime 2} \sin ^{2} \theta_{m}}}$.

Comme $A_{1} C_{1}-B_{1}^{2}=\frac{\tan ^{2} \theta_{m}}{k_{i}^{2}} \frac{\alpha_{0}^{2}+\alpha_{1}^{2}+4 \eta_{m}^{2}}{\alpha_{0}^{2} \alpha_{1}^{2} \eta_{m}^{2}}$, on obtient

$$
V_{i}=r_{m} \pi^{3 / 2} k_{i}^{3} \cot \theta_{m} \frac{\alpha_{0} \alpha_{1} \eta_{m}}{\sqrt{\alpha_{0}^{2}+\alpha_{1}^{2}+4 \eta_{m}^{2}}} \frac{\beta_{0} \beta_{1}}{\sqrt{\beta_{0}^{2}+\beta_{1}^{2}+4 \eta_{m}^{\prime 2} \sin ^{2} \theta_{m}}}
$$

et la quantité équivalente $V_{f}$ pour les neutrons diffusés.

$\mathrm{Si}$ on suppose que tout neutron incident est diffusé de façon complètement aléatoire, l'intégration sur les trajets avant et après l'échantillon correspond au simple produit $V_{i} V_{f}$ qui est donc l'intégrale de la résolution.

\subsubsection{La fonction de résolution}

La fonction de résolution est donnée par l'intégration sur tousles $\vec{k}_{i}$ et $\vec{k}_{f}$ qui respectent les lois de conservation du moment et de l'énergie, ce qui peut s'écrire :

$$
\int \mathcal{P}_{H} \mathcal{P}_{V} \delta\left(\overrightarrow{k_{i}+d k}-\overrightarrow{k_{f}+d k^{\prime}}-\vec{Q}\right) \delta\left(\alpha \omega-\left(\left|\overrightarrow{k_{i}+d \vec{k}}\right|^{2}-\left|\overrightarrow{k_{f}+d k^{\prime}}\right|^{2}\right)\right) k_{i}^{2} k_{f}^{2} \partial d k \partial d k^{\prime} \partial \gamma_{1} \partial \gamma_{2} \partial \delta_{1} \partial \delta_{2}
$$

$\mathrm{Si}$ on prend comme repère l'axe $\vec{x}$ suivant $\vec{Q}_{0}$, l'axe $\vec{y}$ perpendiculaire dans le plan de diffusion horizontal et l'axe $\vec{z}$ vertical, l'angle $\left(\vec{x}, \vec{k}_{i}\right)=\psi$ et l'angle $\left(\vec{k}_{i}, \vec{k}_{f}\right)=\phi$ et qu'on définit $\vec{X} \quad\left\{\begin{array}{l}X_{1}=Q_{x}-Q_{0 x} \\ X_{2}=Q_{y}-Q_{0 y} \\ X_{3}=Q_{z}-Q_{0 z} \\ X_{4}=\omega-\omega_{0}\end{array}\right.$ 
8.1.3 Les relations de conservations du moment et de l'énergie

$\overrightarrow{k_{i}+d k}-\overrightarrow{k_{f}+d k^{\prime}}-\vec{Q}=0$ et $\alpha \omega-\left(\left|\overrightarrow{k_{i}+d k}\right|^{2}-\left|\overrightarrow{k_{f}+d k^{\prime}}\right|^{2}\right)=0$ entraînent les relations :

$$
\left\{\begin{aligned}
X_{1} & =d k \cos \psi-d k^{\prime} \cos (\psi+\phi)-k_{i} \gamma_{1} \sin \psi+k_{f} \gamma_{2} \sin (\psi+\phi) \\
X_{2} & =d k \sin \psi-d k^{\prime} \sin (\psi+\phi)+k_{i} \gamma_{1} \cos \psi-k_{f} \gamma_{2} \cos (\psi+\phi) \\
X_{3} & =k_{i} \delta_{1}-k_{f} \delta_{2} \\
\alpha X_{4} & =2\left(k_{i} d k-k_{f} d k^{\prime}\right)
\end{aligned}\right.
$$

d'où on déduit la résolution verticale. Elle s'écrit :

$\int \mathcal{P}_{V} \partial\left(X_{3}-k_{i} \delta_{1}+k_{f} \delta_{2}\right) \partial \delta_{1} \partial \delta_{2}=\int \frac{\beta_{0} \beta_{3} \exp -\left(D_{1} \delta_{1}^{2}+D_{2} \delta_{2}^{2}\right)}{\sqrt{\left(\beta_{0}^{2}+4 \eta_{m}^{\prime 2} \sin ^{2} \theta_{m}\right)\left(\beta_{3}^{2}+4 \eta_{a}^{\prime 2} \sin ^{2} \theta_{a}\right)}} \partial\left(X_{3}-k_{i} \delta_{1}+k_{f} \delta_{2}\right) \partial \delta_{1} \partial \delta_{2}$

ce qui s'intègre en donnant

$$
R_{V}\left(X_{3}\right)=P \exp -A_{33} X_{3}^{2}
$$

avec

$$
P \frac{\pi \beta_{0} \beta_{3}}{\sqrt{\left(\beta_{0}^{2}+4 \eta_{m}^{\prime 2} \sin ^{2} \theta_{m}\right)\left(\beta_{3}^{2}+4 \eta_{a}^{\prime 2} \sin ^{2} \theta_{a}\right)}} \frac{\sqrt{\pi}}{\sqrt{D_{1} k_{f}^{2}+D_{2} k_{i}^{2}}}
$$

et $A_{33}=D_{1} D_{2} /\left(D_{1} k_{f}^{2}+D_{2} k_{i}^{2}\right)$.

Pour la résolution horizontale, nous introduisons les vecteurs à 4 dimensions $\vec{Y}\left(d k, d k^{\prime}, k_{i} \gamma_{1}, k_{f} \gamma_{2}\right)$ et $\vec{t}\left(X_{1}, X_{2}, X_{4}, k_{f} \gamma_{2}\right)$ La composante horizontale de la résolution s'écrit alors

$$
R_{H}=\int \exp -\vec{Y}^{t} \mathbf{S} \vec{Y} \delta_{3}(\vec{t}-\mathbf{M} \vec{Y}) k_{i} k_{f} \partial^{4} Y
$$

où $\delta_{3}$ signifie que la fonction $\delta$ ne joue que sur les 3 premières composantes de $\vec{t}$ et où on a défini

$$
\mathbf{S}=\left(\begin{array}{cccc}
A_{1} & 0 & B_{1} / k_{i} & 0 \\
0 & A_{2} & 0 & B_{2} / k_{f} \\
B_{1} / k_{i} & 0 & C_{1} / k_{i}^{2} & 0 \\
0 & B_{2} / k_{f} & 0 & C_{2} / k_{f}^{2}
\end{array}\right) \text { et } \mathbf{M}=\left(\begin{array}{cccc}
\cos \psi & -\sin \psi & -\cos (\psi+\phi) & \sin (\psi+\phi) \\
\sin \psi & \cos \psi & -\sin (\psi+\phi) & -\cos (\psi+\phi) \\
2 k_{i} / \alpha & 0 & -2 k_{f} / \alpha & 0 \\
0 & 0 & 0 & 1
\end{array}\right)
$$

L'intégration sur $Y_{1}, Y_{2}, Y_{3}$ donne

$$
R_{H}=\frac{k_{i} k_{f}}{|\operatorname{det} \mathbf{M}|} \int \exp -\vec{t}^{t} \mathbf{B} \vec{t} \partial Y_{4}
$$

avec $\operatorname{det} \mathbf{M}=\frac{2}{\alpha}\left(k_{f}-k_{i} \cos \phi\right)^{-1}$ et $\mathbf{B}=\mathbf{M}^{-1}{ }^{t} \mathbf{S} \mathbf{M}^{-1}$.

En développant

$$
R_{H}=\frac{\alpha k_{i} k_{f}}{2\left|k_{f}-k_{i} \cos \phi\right|} \int \exp -\left(\Sigma_{i, j=1,3} Y_{i} B_{i j} Y_{j}+2 \Sigma_{i=1,3} Y_{i} B_{i 4} Y_{4}+B_{44} Y_{4}^{2}\right) \partial Y_{4}
$$

on voit que si on définit $A_{i j}^{\prime}=B_{i j}-B_{i 4} B_{j 4} / B_{44}$ pour $i$ et $j=1,2,3$ correspondant à $X_{1}, X_{2}, X_{4}$, qu'on s'empressera de redéfinir $A_{i j}=A_{k l}^{\prime}$ avec $i$ ou $j=4$ pour $k$ ou $l=3$, et $A_{i 3}=0 \quad \forall i$ et qu'on regroupe avec la composante verticale on arrive à l'écriture

$$
R=R_{0} \exp -\Sigma_{i, j=1,4} X_{i} A_{i j} X_{j}=R_{0} \exp -\vec{X}^{t} \mathbf{A} \vec{X}
$$

L'intégration sur $X_{1}, X_{2}, X_{3}, X_{4}$ donne $N=\pi^{2} R_{0} / \sqrt{\operatorname{det} \mathbf{A}}=V_{i} V_{f} \operatorname{donc} R_{0}=V_{i} V_{f} \sqrt{\operatorname{det} \mathbf{A}} / \pi^{2}$.

L'avantage de la formulation Cooper-Nathans est qu'elle permet d'avoir facilement des repères qualitatifs, en définissant, si nécessaire, des collimations effectives sur les trajets où il n'y a pas de 
collimateurs à lames (typiquement $\left(L_{1}+L_{2}\right) / 2 D$ pour 2 éléments où $L_{1}$ et $L_{2}$ sont les largeur projetées sur le plan perpendiculaire à la trajectoire et $D$ la distance qui les sépare).

Exemple : La largeur(fwhm) en énergie d'un mode non dispersif est donnée par

$$
\Delta \omega=\frac{2}{\alpha}\left[\frac{h_{0}^{2} h_{1}^{2}+\eta_{m}^{2}\left(h_{0}^{2}+h_{1}^{2}\right)}{h_{0}^{2}+h_{1}^{2}+4 \eta_{m}^{2}} k_{i}^{4} \cot ^{2} \theta_{m}+\frac{h_{2}^{2} h_{3}^{2}+\eta_{a}^{2}\left(h_{2}^{2}+h_{3}^{2}\right)}{h_{2}^{2}+h_{3}^{2}+4 \eta_{a}^{2}} k_{f}^{4} \cot ^{2} \theta_{a}\right]^{\frac{1}{2}}
$$

si $h_{0}, h_{1}, h_{2}, h_{3}$ sont les collimations horizontales(fwhm) et $\eta_{m}, \eta_{a}$ les mosaïcités(fwhm) du monochromateur et de l'analyseur.

Attention ! il ne faut pas oublier que la collimation naturelle peut devenir inférieure à celle imposée par un collimateur à lames. En particulier les surfaces projetées du monochromateur et de l'analysuer varient en $\sin \theta_{m}$ et $\sin \theta_{a}$.

REMARQUE : Le calcul présenté ci-dessus a éludé la gestion des signes liés à la configuration du spectromètre. Pour chaque axe - monochromateur, échantillon, analyseur - il faut associer un signe fonction du sens de rotation (clockwise ou anticlockwise). Ces signes interviennent en particulier pour la focalisation évoquée en section 6.1 .

Pour aller au-delà il faut prendre en compte la géométrie réelle du spectromètre: dimension de la source et du détecteur, dimension et courbure des cristaux, distances entre éléments et diaphragmes limitant le faisceau.

\subsection{Prise en compte de la géométrie du spectromètre : approximation gaussienne}

L'étape suivante consiste à prendre en compte les effets liés à la dimension finie de la source et des cristaux, y compris l'échantillon, à la présence éventuelle de diaphragmes sur le faisceau et à l'utilisation des courbures. Il faut donc introduire les coordonnées spatiales du neutron. La taille finie des éléments correspond à une fonction "créneau" et les courbures induisent de nouvelles corrélations. La solution complète ne peut alors être obtenue que par des méthodes numériques. On peut se simplifier la vie en remplaçant le créneau par une approximation gaussienne et en développant au premier ordre les effets liés aux courbures, comme indiqué en section 4.2. On conserve ainsi une description analytique de la fonction de résolution, ce qui permet une simplification notable des calculs de simulation et d'analyse des résultats.

C'est cette étape qui a été utilisée pour les simulations présentées dans cet article. Elle permet également une analyse rapide et efficace au fur et à mesure de l'acquisition des résultats en cours de manip. L'expérience acquise démontre que cette étape est suffisante dans la plupart des cas pour prendre en compte les biais des mesures signalés dans la section 6 .

\section{ANALYSE DES RÉSULTATS}

La mesure sur spectromètre 3-axes se fait dans un espace à 4 dimensions. Pour extraire les paramètres physiques d'intérêt, il faut prendre en compte la distorsion introduite par la fonction de résolution. La statistique de comptage, généralement faible, n'est guère propice à l'utilisation de méthodes de déconvolution. Le niveau de complexité de l'analyse des résultats doit être adapté à l'objectif scientifique visé. Dans de nombreux cas, il s'agit d'obtenir une bonne définition d'une courbe de dispersion, de déterminer la présence d'un amortissement et, dans l'affirmative, sa dépendance en q, puis de suivre l'évolution du système en fonction d'un paramètre extérieur, température, pression, champ magnétique, etc.... Généralement dans les installations qui disposent de spectromètres 3-axes ont été développés des logiciels de traitement de données. Renseignez-vous près de votre correspondant local pour voir s'il y a des outils adaptés à vos besoins. 
Si on souhaite un outil simple et rapide, on peut utiliser l'approximation gaussienne de la fonction de résolution et une fonction de diffusion un peu simplifiée pour obtenir un dépouillement rapide en cours d'expérience et avoir ainsi une meilleure maîtrise de la stratégie de mesure. En voici le principe.

\subsection{Méthode simplifiée : cas sans amortissement}

En l'absence d'amortissement, on peut utiliser la méthode proposée par Haywood [3] qui consiste à utiliser une représentation intégrale de la fonction de Dirac: $\delta(x)=\frac{1}{2 \pi} \int \partial \mu \exp -i \mu x$.

Si la nappe de dispersion, au voisinage du point de mesure, peut être décrite par une équation du second degré $\vec{X}^{t} \mathbf{B} \vec{X}+\overrightarrow{\mathrm{T}}^{t} \vec{X}+H=0$, avec $X_{i=1,3}=\left(Q-Q_{0}\right)_{i=1,3}, X_{4}=\omega-\omega_{0}$, et $\vec{Q}=\tau+\vec{q}$. L'intensité peut alors s'écrire:

$$
I^{\prime}\left(\vec{Q}_{0}, \omega_{0}\right)=N_{Q_{0}} \frac{R_{0}}{2 \pi} \int \partial \mu \int \partial^{4} X \exp -\vec{X}^{t} \mathbf{A} \vec{X}-i \mu\left[\left(\vec{X}+\vec{X}_{0}\right)^{t} \mathbf{B}\left(\vec{X}+\vec{X}_{0}\right)+\vec{T} \cdot\left(\vec{X}+\vec{X}_{0}\right)+H\right]
$$

où $N_{Q}$ contient le facteur de population thermique, le facteur de structure fonction de $\vec{Q}$ et un facteur d'échelle fonction du volume de l'échantillon et de la puissance du réacteur. On peut généralement supposé $N_{Q}$ peu dépendant dans le domaine d'intégration et l'assimiler à $N_{Q_{0}}$.

ATTENTION : Le repère utilisé ici est un repère orthonormé, où l'axe $X_{1}$ correspond à l'axe $i_{1} a^{*}+i_{2} b^{*}+i_{3} c^{*}$ qui définit l'axe $x$ du plan de diffusion, les axes $X_{2}$ et $X_{3}$ complétant le repère, $X_{2}$ perpendiculaire à $X_{1}$ dans le sens trigonométrique, le tout en unité $\AA^{-1}$.

Ceci permet une intégration analytique sur $\overrightarrow{\mathbf{X}}$ qui ramène le calcul de l'intensité à une intégration numérique à une dimension:

$$
I^{\prime}\left(\vec{Q}_{0}, \omega_{0}\right)=N_{Q_{0}} \frac{R_{0}}{2 \pi} \int \partial \mu \frac{\pi^{2}}{\sqrt{\operatorname{det}(\mathbf{C}(\mu))}} \exp -\left(i \mu K+\mu^{2} \vec{D}^{t} \mathbf{C}^{-1} \vec{D}\right)
$$

avec $\mathbf{C}=\mathbf{A}+i \mu \mathbf{B}, \vec{D}=\mathbf{B} \vec{X}_{0}+\frac{1}{2} \vec{T}$ et $K=\vec{X}_{0}^{t} \mathbf{B} \vec{X}_{0}+\vec{T}^{t} \cdot \vec{X}_{0}+H$

Dans le cas d'une dispersion linéaire $\mathbf{B}=\mathbf{0}$, l'intégration analytique donne, en posant $f\left(\vec{Q}_{0}\right)-$ $\omega_{0}=\vec{T}^{t} \cdot \vec{X}_{0}+H$ et $\sigma=\vec{T}^{t} \mathbf{A}^{-1} \vec{T}:$

$$
I^{\prime}\left(\vec{Q}_{0}, \omega_{0}\right)=N_{Q_{0}} \frac{R_{0} \pi^{2}}{\sqrt{\operatorname{det}(\mathbf{A})}} \frac{1}{\sqrt{\pi} \sigma} \exp -\frac{\left[\omega_{0}-f\left(\vec{Q}_{0}\right)\right]^{2}}{\sigma^{2}}=N_{Q_{0}} V_{I} \cdot V_{F} \frac{1}{\sqrt{\pi} \sigma} \exp -\frac{\left[\omega_{0}-f\left(\vec{Q}_{0}\right)\right]^{2}}{\sigma^{2}}
$$

Dans le cas non-linéaire, il reste une intégration à une dimension qui se fait numériquement sans problème, à condition d'assurer la continuité de la phase dans le calcul de $\sqrt{\operatorname{det}(\mathbf{C}(\mu))}$.

\subsection{Méthode simplifiée : cas avec amortissement}

Pour profiter de la méthode de calcul rapide du cas sans amortissement, on cherche à assimiler l'effet de l'amortissement à l'existence d'une distribution de courbes non amorties, pour aboutir à un calcul approché de la forme $I^{\prime}\left(\vec{Q}_{0}, \omega_{0}\right)=\int_{0}^{\infty} \partial \alpha P(\alpha) I_{\alpha}\left(\vec{Q}_{0}, \omega_{0}\right)$ où $I_{\alpha}\left(\vec{Q}_{0}, \omega_{0}\right)$ est le calcul sans amortissement pour une courbe de la forme $\omega=\alpha f(\vec{q})$ ou $\omega^{2}=\alpha^{2} f(\vec{q}), f(\vec{q})$ restant une forme bilinéaire en $\vec{q}$.

$$
\begin{gathered}
\mathrm{D}^{\prime} \mathrm{o} I_{\alpha}\left(\vec{Q}_{0}, \omega_{0}\right)=N_{Q_{0}} \frac{1}{2 \pi} \int \partial \mu \int \partial^{3} Q \partial \omega R\left(\vec{Q}-\vec{Q}_{0}, \omega-\omega_{0}\right) \exp -i \mu(\omega-\alpha f(\vec{Q})) \\
\text { ou } I_{\alpha}\left(\vec{Q}_{0}, \omega_{0}\right)=N_{Q_{0}} \frac{1}{2 \pi} \int \partial \mu \int \partial^{3} Q \partial \omega R\left(\vec{Q}-\vec{Q}_{0}, \omega-\omega_{0}\right) 2 \alpha \sqrt{f(\vec{Q})} \exp -i \mu\left(\omega^{2}-\alpha^{2} f(\vec{Q})\right)
\end{gathered}
$$

Le choix de la famille de courbes est bien sûr fonction de la physique étudiée. Pour que la méthode soit valable, il est impératif que la pondération $P(\alpha)$ dépende uniquement de $\vec{Q}_{0}$ et $\omega_{0}$. Il faut donc choisir 
les approximations les plus adaptées à la mesure qu'on analyse. En utilisant une méthode d'intégration qui utilise des valeurs tabulées (méthode de Gauss), on aboutit à

$$
I^{\prime}\left(\vec{Q}_{0}, \omega_{0}\right)=\sum_{i} c_{i} P\left(\alpha_{i}\right) I_{\alpha_{i}}\left(\vec{Q}_{0}, \omega_{0}\right)
$$

où les valeurs de $\alpha_{i}$ et les poids $c_{i}$ sont définis par $\mathbf{n}$, l'ordre du développement de Gauss.

Par ce biais, les paramètres physiques à extraire de la mesure apparaissent uniquement dans $P(\alpha)$. Il suffit de calculer une seule fois la "grille" de $I_{\alpha_{i}}\left(\vec{Q}_{0}, \omega_{0}\right)$, ce qui fournit un calcul très rapide, moyennant des approximations raisonnables pour obtenir une bonne estimation des paramètres recherchés.

\subsection{Méthode générale}

Dans les cas où une méthode simplifiée n'est pas applicable, il ne reste que l'intégration numérique à quatre dimensions. Suivant qu'il s'agit d'une simulation ou d'une analyse de résultats, le point clé restera la précision numérique à atteindre pour éviter les artefacts numériques. L'approximation gaussienne de la fonction de résolution permet un gain de temps appréciable.

\section{CONCLUSION - REMARQUES SUR LES AUTRES TECHNIQUES NEUTRONIQUES POUR LES MESURES SUR MONOCRISTAUX}

La section efficace de diffusion inélastique des neutrons est faible et les réacteurs de recherche à flux continu sont proches de leur optimum. Pour diminuer le temps de mesure et pour avoir la possibilité d'obtenir des résultats sur des monocristaux de faible volume, le spectromètre 3-axes a progressivement évolué pour optimiser l'usage des faisceaux de neutrons. L'utilisation des courbures du monochromateur et de l'analyseur, une augmentation de la taille des faisceaux, l'utilisation de miroirs pour guider les faisceaux ont permis de gagner près de 2 ordres de grandeur dans les 20 dernières années. Le volume minimum d'échantillon se compte maintenant en $\mathrm{mm}^{3}$ et même moins dans les cas favorables.

Deux questions se posent actuellement :

- peut-on faire mieux sur une source à flux continu ?

- que peut-on espérer d'une source pulsée?

Dans les deux cas, la question concerne l'utilisation de compteurs multiples pour cumuler l'information. Le débat est loin d'être clos et des tentatives diverses sont en cours d'évaluation. Voici les éléments qu'il faut prendre en compte pour essayer de faire des choix réalistes.

- La mesure pas à pas du spectromètre 3-axes classique permet de sélectionner spécifiquement la portion d'espace réciproque que l'on veut explorer et la direction précise du vecteur de diffusion. L'optimisation de l'utilisation du faisceau permet un gain d'intensité pour chaque pas de la mesure.

- La mesure avec des détecteurs multiples, quel que soit le type de source, permet l'acquisition simultanée sur une large portion de l'espace réciproque. L'échantillon étant fixe, les règles de conservation du moment et de l'énergie définissent cette portion d'espace. Il est donc généralement nécessaire de répéter l'acquisition avec des positions différentes de l'échantillon pour avoir un ensemble plus complet et pour sélectionner un sous-ensemble particulier. Les techniques d'optimisation du flux ne sont plus applicables. L'intensité par cellule élémentaire est donc plus faible.

- Les sources pulsées permettent l'installation d'un nombre considérables de cellules de détection (40000 sur maps à Isis). L'intensité par cellule est malgré tout fonction du flux moyen de la source et non du flux au pic. 
Les mesures faites actuellement à Isis et les essais réalisés à l'ILL permettent quelques réflexions:

- Dans l'état actuel des sources pulsées, on obtient de bons résultats avec des volumes d'échantillon généralement de plusieurs $\mathrm{cm}^{3}$. L'acquisition automatique dans une large portion de l'espace réciproque a permis de révéler des singularités, en particulier dans le domaine des supraconducteurs à haute température critique, qui auraient sans doute échappé aux mesures sur spectromètre 3 -axes classique.

- L'analyse fine de ces singularités n'a pu être réalisée que sur spectromètre 3-axes car elle nécessitait une bonne intensité dans la région d'intérêt et un suivi en température. Sur une source pulsée, pour chaque changement de paramètre (température, champ magnétique, pression, etc...), il faut répéter l'acquisition complète.

- Les essais sur $\mathrm{CuGeO}_{3}$ faits à l'ILL sur une extension de type flat-cone sont peu convaincants. Le gain de temps ne compense pas la diminution du rapport signal/bruit et les caractéristiques essentielles de la diffusion magnétique en dessous de la transition spin-Peierls ont été quelque peu ratées...

En conclusion, on peut avec prudence affirmer que le développement des sources pulsées, SNS aux étatsunis, ESS peut-être dans un futur proche en Europe, permettront d'obtenir des résultats similaires à ceux d'Isis, avec un facteur d'échelle intéressant. Sauf malheureusement dans le domaine des monocristaux de petit volume. La capacité de mesures très fines et dépendant d'un paramètre extérieur restera l'apanage des appareils sur source continue, de même que la possibilité d'obtenir des résultats de qualité sur des monocristaux de faible volume. Il est souhaitable que les installations existantes gardent ces objectifs dans leur priorité.

\section{Références}

Quelques références générales :

M.J. Cooper and R. Nathans Acta Crystallogr. 23, 357 (1967)

B. Dorner Acta Crystallogr. A28, 319 (1972)

B.C. Haywood Acta Crystallogr. A27, 408 (1971) 